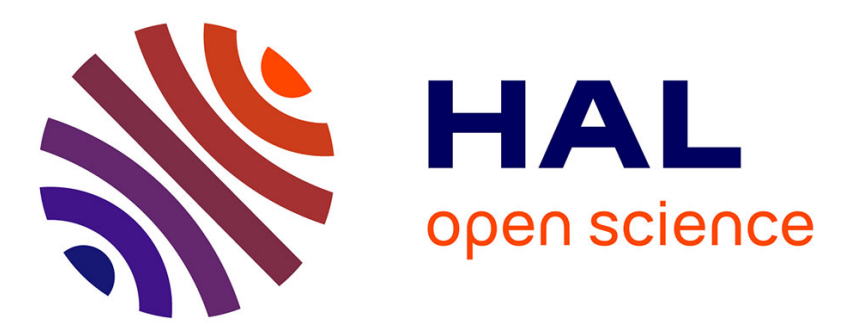

\title{
Solutions of polydiacetylenes in good and poor solvents : a light and neutron scattering study
}

\author{
M. Rawiso, J.P. Aime, J.L. Fave, M. Schott, M.A. Müller, M. Schmidt, H. \\ Baumgartl, G. Wegner
}

\section{To cite this version:}

M. Rawiso, J.P. Aime, J.L. Fave, M. Schott, M.A. Müller, et al.. Solutions of polydiacetylenes in good and poor solvents: a light and neutron scattering study. Journal de Physique, 1988, 49 (5), pp.861-880. 10.1051/jphys:01988004905086100 . jpa-00210763

\section{HAL Id: jpa-00210763 https://hal.science/jpa-00210763}

Submitted on 1 Jan 1988

HAL is a multi-disciplinary open access archive for the deposit and dissemination of scientific research documents, whether they are published or not. The documents may come from teaching and research institutions in France or abroad, or from public or private research centers.
L'archive ouverte pluridisciplinaire HAL, est destinée au dépôt et à la diffusion de documents scientifiques de niveau recherche, publiés ou non, émanant des établissements d'enseignement et de recherche français ou étrangers, des laboratoires publics ou privés. 


\title{
Solutions of polydiacetylenes in good and poor solvents : a light and neutron scattering study
}

\author{
M. Rawiso $\left({ }^{1, *}\right)$, J. P. Aime $\left({ }^{2}\right)$, J. L. Fave $\left({ }^{2}\right)$, M. Schott $\left({ }^{2}\right)$, M. A. Müller $\left({ }^{3, * *}\right)$, \\ M. Schmidt $\left({ }^{3}\right)$, H. Baumgartl $\left({ }^{3}\right)$ and G. Wegner $\left({ }^{3}\right)$ \\ (1) Institut Laue Langevin, B.P. 156X, 38042 Grenoble Cedex, France \\ $\left({ }^{2}\right)$ Groupe de Physique des Solides de l'ENS, UA CNRS 17, Université Paris VII, 2 place Jussieu, 75251 \\ Paris Cedex 05, France \\ ( $\left.{ }^{3}\right)$ Max-Planck Institut für Polymerforschung, Jakob Welder Weg 11, 6500 Mainz, F.R.G.
}

(Reçu le 10 août 1987, révisé le 2 février 1988, accepté le 2 février 1988)

\begin{abstract}
Résumé. - On a étudié les propriétés de solutions de P-3BCMU et P-4BCMU en bons et mauvais solvants par des techniques de diffusion de lumière et de neutrons. En bon solvant, les solutions sont jaunes et les chaînes de $\mathrm{P}-3 \mathrm{BCMU}$ et $\mathrm{P}-4 \mathrm{BCMU}$ sont des pelotes semi-rigides dans l'état isomérique trans. Leur structure interne est mieux décrite par un modèle continu de ruban semi-rigide que par un modèle de défauts discrets séparant des plaquettes de longueurs variables et d'orientations différentes. A la température ambiante, et pour les deux polydiacétylènes, on trouve une longueur de persistance longitudinale proche de $160 \AA$, et un carré moyen du rayon de gyration axial, relié à l'extension latérale des macromolécules, de l'ordre de $60 \AA^{2}$. La rigidité du squelette conjugué ne semble pas dépendre de la présence ou non de liaisons hydrogène entre groupes latéraux voisins, tant que ces liaisons restent en proportion assez faible. En mauvais solvant, et spécifiquement pour des solutions rouges de $\mathrm{P}-4 \mathrm{BCMU}$ dans le toluène, les chaînes forment des agrégats complexes de masse moléculaire élevée. On n'a pas observé de véritables solutions rouges de bâtonnets isolés, même à des concentrations aussi faibles que $0,5 \times 10^{-3} \mathrm{~g} \mathrm{l}^{-1}$ de $\mathrm{P}-4 \mathrm{BCMU}$ de grande masse moléculaire $\left(M_{\mathrm{w}}=1,67 \times 10^{6} \mathrm{gmole}^{-1}\right)$ ou $0,5 \mathrm{gl}^{-1}$ de $\mathrm{P}-4 \mathrm{BCMU}$ de petite masse moléculaire $\left(M_{\mathrm{w}}=4,32 \times 10^{4} \mathrm{~g}\right.$ mole $\left.{ }^{-1}\right)$. Ceci suggère que dans toutes les expériences sur les solutions bleues ou rouges de $\mathrm{P}-3 \mathrm{BCMU}$ et $\mathrm{P}$ 4BCMU on n'observe que des agrégats. Cependant, la question reste ouverte de savoir si les modifications de la structure électronique observées sont dues à une agrégation et/ou une cristallisation partielle, ou si elles proviennent d'une transition pelote-bâtonnet suivie d'une agrégation subséquente des bâtonnets. Finalement, on discute le processus d'agrégation tel qu'il est observé par diffusion des neutrons aux petits angles.
\end{abstract}

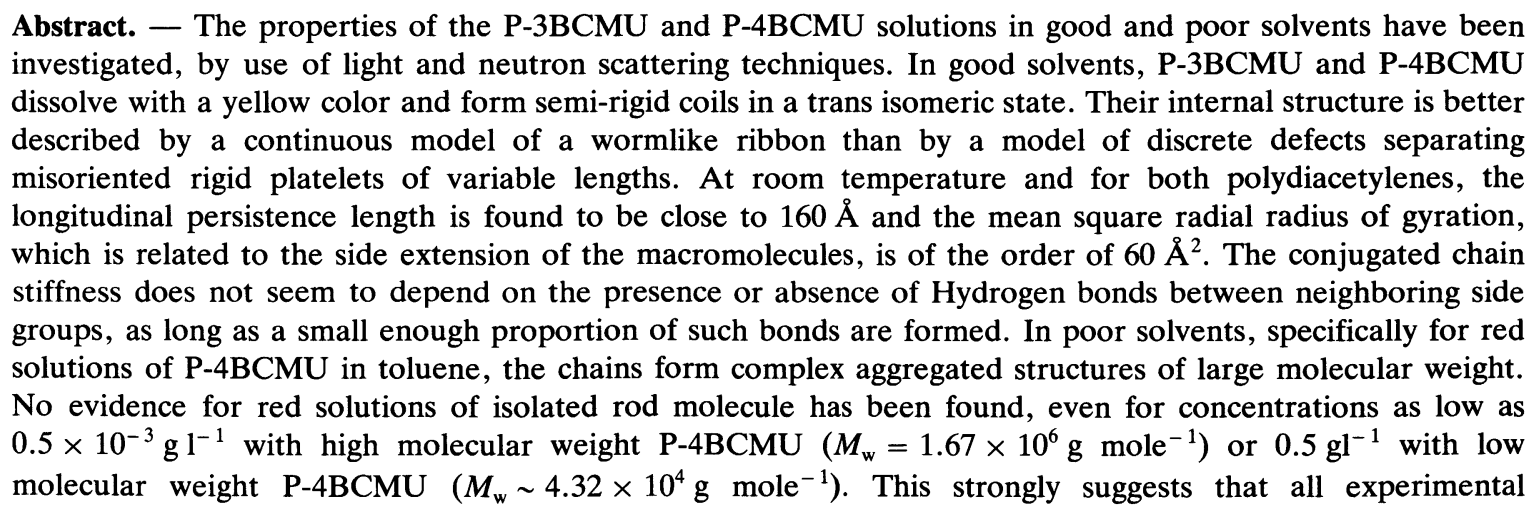

(*) Present address : Laboratoire de Physique des Solides, Bât. 510, Université Paris Sud, 91405 Orsay, France.

(**) Present address : Röhm GmbH, 6100 Darmstadt, F.R.G. 
investigations on the blue and red solutions of P-3BCMU and P-4BCMU observe the aggregated structures only. However, it still remains an open question whether the observed change in the electronic structure is caused by aggregation and/or partial crystallization or whether the color change originates from an instantaneous coil to rod transition followed by subsequent aggregation of the rods. The aggregation process, as seen by small angle neutron scattering, is studied and discussed.

\section{Introduction.}

Polydiacetylenes are linear polymers with general formula $\quad(\mathrm{CR}=\mathrm{CR}-\mathrm{C} \equiv \mathrm{C})_{n}$. A few polydiacetylenes, with large and suitable side groups $R$, are soluble in organic solvents [1-3]. Since their backbone contains conjugated double and triple bonds, they give an opportunity to investigate the coupling of the electronic structure with the average conformation, bearing on the problem of electron localization in one-dimensional systems in the presence of diagonal and non-diagonal disorder. They are also good candidates for studying semi-rigid macromolecules.

Poly-3 and 4BCMU belong to this class of polydiacetylenes and $R$ stands for 3- and 4butoxycarbonyl methylurethane, i.e. :

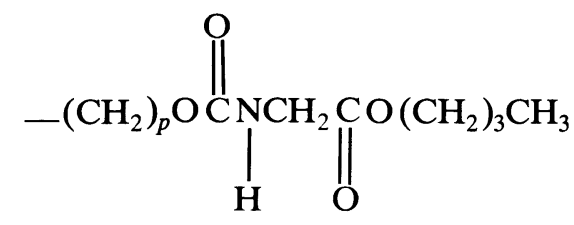

with

$$
p=3 \text { and } 4 \text { respectively } .
$$

In good solvents, they form yellow solutions, and a drastic color change is observed as the solvent quality is reduced. For instance, when hexane is added to a solution of P-3BCMU in chloroform at room temperature, it turns blue [4]; and when a solution of $\mathrm{P}-4 \mathrm{BCMU}$ in toluene is cooled from high temperature, it turns red [5]. These color transitions correspond to changes in the electronic structure of the carbon chain skeleton. The study of the related conformational modifications is a subject of current interest.

It is accepted that these polymers form coils in their yellow solutions, but the internal structure of these coils is a matter of controversy. From light scattering experiments, a wormlike chain model, leading to a ribbon like structure when the spatial resolution is increased, has been proposed $[2,6]$. In this continuous model, the stiffness of the chain is mainly due to the conjugated structure of the backbone. However a model of misoriented rigid platelets of variable length was preferred to explain light absorption experiments $[4,7,8]$. The planar structure of each segment is then reinforced by hydrogen bonds between adjacent side groups on the same side of the chain within a platelet, and rotations around $\sigma$ bonds in the carbon chain skeleton are supposed to interrupt the conjugation as well as hydrogen bonding. In this discrete model, the planar structure is lost locally through defects. On the other hand, it is generally believed that the conjugated backbone is in the trans configuration of the double bonds, but it has been suggested that yellow solutions consist primarily of the cis configuration [9]. Since polydiacetylenes have always been found to be trans in the solid state, the yellow to blue (or red) transition might then correspond to a cis $\rightarrow$ trans isomerization.

For color transitions, two main structural models have been given. The first one, similar to the model of Zimm and Bragg for the helix $\rightarrow$ coil transition [10], is related to pure intramolecular coil to rod conformational changes $[5,11-13]$ which may or may not be connected to a cis $\rightarrow$ trans isomeric modification and corresponds to the formation of the highest possible number of hydrogen bonds. Beyond a critical polymer concentration, gelation occurs, associated with an overlap of rods, following a percolation process $[14,15]$. The second model corresponds to an aggregation of semi-rigid chains, or parts of such chains, leading to clusters $[6,16]$ : this is the fringed micelles model [17].

The present study aims at clarifying these questions. It describes light and neutron scattering experiments which provide information about structures of P-3 and 4BCMU solutions at two distinct spatial scales. Experimental details are given in section 2, while results are presented and discussed from section 3 to 5 . The average conformation of high molecular weight P-3 and 4BCMU chains in good solvents is first studied in section 3. The main features of the color transition for P-4BCMU solutions in toluene are described in section 4. For high molecular weight polymers, and typical polymer concentrations needed for neutron scattering experiments, it is shown that aggregation occurs. The question therefore arises of the existence of a true red solution. A light scattering study of very dilute solutions of high molecular weight polymers is then described in section 5. Neutron scattering experiments are also reported in the same section, for lower molecular weight polymers. 


\section{Experimental procedures.}

2.1 SAMPLES. - The monomers 3 and 4BCMU were synthesized and crystallized as described in the literature [1, 17]. The polymers P-3 and 4BCMU were then obtained by exposure to ${ }^{60} \mathrm{Co}-\gamma$-radiation of single crystals of monomers, at $T=30^{\circ} \mathrm{C}$. The total dose was $3 \mathrm{Mrad}$, leading to $36 \%$ polymer conversion for $3 \mathrm{BCMU}$ and $67 \%$ for $4 \mathrm{BCMU}$. The unreacted monomers were extracted with acetone and di-isopropylether for 3 and 4BCMU, respectively.

In this way, high molecular weight polymers were prepared. Their average molecular weight $M_{\mathrm{w}}$ and average radius of gyration $\left\langle R_{\mathrm{G}}^{2}\right\rangle_{z}^{1 / 2}$, where \langle\rangle$_{z}$ refers to the $z$-average on the molecular weight distribution, were measured by light scattering experiments from dilute solutions in chloroform [6, 17]. They provide an estimation of the overlap concentrations defining regions of cross-over between dilute and semi-dilute regimes:

$$
c^{*}=\frac{M_{\mathrm{w}}}{\mathcal{N} R_{\mathrm{G}}^{3}}
$$

where $\mathcal{N}$ is Avogadro's number. The molecular weight distributions were determined by gel permeation chromatography. The solvent was chloroform and the different average molecular weights $M_{\mathrm{N}}$, $M_{\mathrm{w}}, M_{z}$ were derived from the calibration curve established previously for another polydiacetylene, P-TS12 [2] which is very similar to the polystyrene calibration curve.

Low molecular weight polymers of P-4BCMU were also prepared by ultrasonication of dilute solutions in DMF of the high molecular weight sample referred to as P-4BCMU-1 in table I. The average molecular weight $M_{\mathrm{w}}$ and average radius of gyration $\left[\left\langle R_{\mathrm{G}}^{2}\right\rangle_{z}\right]^{1 / 2}$ were obtained by light as well as neutron scattering experiments. The polydispersity was found comparable to the source sample P4BCMU-1 polydispersity, as expected since sonication produces roughly local shear gradients causing

Table I. - Characteristics of $P-3$ and $4 B C M U$ samples.

\begin{tabular}{|c|c|c|c|}
\hline Sample & $\begin{array}{c}M_{\mathrm{w}} \\
\left(\mathrm{g} \mathrm{mole}^{-1}\right)\end{array}$ & $\begin{array}{c}U= \\
\left(M_{\mathrm{w}} / M_{\mathrm{N}}\right)-1\end{array}$ & $c^{*}\left(\mathrm{gl}^{-1}\right)$ \\
\hline P-3BMCU-1 & 1360000 & 8 & $\sim 2.72$ \\
\hline P-4BCMU-1 & 1670000 & 1.5 & $\sim 1.82$ \\
\hline P-4BCMU-2 & $\sim 43200$ & & $\sim 40.50$ \\
\hline
\end{tabular}

midpoint scissions of macromolecules with molecular weights higher than a critical value, as in well characterized extensional or turbulent flows [18-20]. One does not expect that sonication will affect the backbone conjugated structure, nor break a significant number of side groups.

Table I lists the main characteristics of the samples used in this study.

2.2 NeUtron SCATTERING EXPERIMENTS. - Small angle neutron scattering experiments were performed on D11 and D17 diffractometers at I.L.L. in Grenoble, France. The range of scattering vectors covered, the spread in the wavelength of the incident neutrons $\Delta \lambda / \lambda$ and the resolution $\Delta q$ due to the divergence of the incident beam are given in table II, for each spectrometer. In the static approximation, $q$ is defined by :

$$
q=\frac{4 \pi}{\lambda} \sin \frac{\theta}{2}
$$

where $\lambda$ is the neutron wavelength and $\theta$, the scattering angle.

Table II. - Characteristics of the neutron scattering geometries.

\begin{tabular}{|c|c|c|c|}
\hline $\begin{array}{c}\text { Spectro- } \\
\text { meter }\end{array}$ & $\begin{array}{c}q\left(\AA^{-1}\right)- \\
\text { range }\end{array}$ & $\begin{array}{c}\Delta \lambda / \lambda \\
(\%)\end{array}$ & $\begin{array}{c}\Delta q \\
\left(10^{-3} \AA^{-1}\right)\end{array}$ \\
\hline $\mathrm{D} 11$ & $0.003<q<0.04$ & 8 & 2 \\
\hline $\mathrm{D} 17$ & $0.008<q<0.15$ & 10 & 4 \\
\hline
\end{tabular}

Several polymer solutions were investigated. Yellow solutions of $\mathrm{P}-3 \mathrm{BCMU}$ in perdeuteriated DMF - four concentrations between 0.7 and $5 \mathrm{gl}^{-1}$ were prepared at $T=85^{\circ} \mathrm{C}$ and studied at room temperature. The $c=1 \mathrm{gl}^{-1}$ solution was also studied at $60^{\circ} \mathrm{C}$. Several solutions of P-4BCMU-1 were prepared : a $1 \mathrm{gl}^{-1}$ solution in perdeuteriated DMF was studied at $60{ }^{\circ} \mathrm{C}$; two solutions in $\mathrm{CDBr}_{3}$, having $c=3$ and $6 \mathrm{gl}^{-1}$, were studied at room temperature. Two solutions in perdeuteriated toluene were prepared at $T=85^{\circ} \mathrm{C}$. In both, $0.4 \%$ of distilled triethylamine was added in order to avoid thermal degradation [21]. The first solution, $c=4.3 \mathrm{gl}^{-1}$, was studied as a function of temperature from $80^{\circ} \mathrm{C}$ to $38^{\circ} \mathrm{C}$, spanning the yellow and red phases. The second, $c=1.2 \mathrm{gl}^{-1}$, was investigated in the yellow phase, for $T \geqslant 67^{\circ} \mathrm{C}$. Finally, two solutions (concentrations : 0.47 and $1 \mathrm{gl}^{-1}$ ) of the low molecular weight P-4BCMU in perdeuteriated toluene were studied at two temperatures : $70.5^{\circ} \mathrm{C}$ and $40.5^{\circ} \mathrm{C}$, corresponding to the yellow and red phases, respectively. 
Sample holders were $5 \mathrm{~mm}$ thick quartz cells. $\mathrm{H}_{2} \mathrm{O}$ standards were studied in $1 \mathrm{~mm}$ thick quartz cells. A $2.5 \mathrm{~mm}$ thick quartz cell was also used for one of the samples, leading to no perceptible difference in the data when normalized to sample thickness. Multiple scattering effects were therefore negligible as indicated also from the ratios of the transmission factors of the solutions and their related solvents, which are close to unity.

The data were treated according to current procedures for small angle isotropic neutron scattering. They were put on an absolute scale by using $\mathrm{H}_{2} \mathrm{O}$ as a standard. Indeed the differential cross section per unit volume of $\mathrm{H}_{2} \mathrm{O}$ is known, at room temperature and for different wavelengths, from instrumental calibrations performed using several well characterized samples [22]. After careful background substractions, the coherent differential cross sections per unit volume $\Sigma^{\mathrm{coh}}(q)\left(\mathrm{cm}^{-1}\right)$ were obtained. The scattering length densities at room temperature involved in this study are given in figure 1. For polymers, they were evaluated assuming additivity for the molar volumes of elementary units like $-\mathrm{CH}_{2}$-, obtained from the literature [23] ; they are not constant within the cross section of the chains. However, at low enough resolution, the side groups can be considered as nearly homogeneous particles in solvents like $\mathrm{DMFD}_{7}$ or perdeuteriated toluene. The contrast factors between macromolecules and

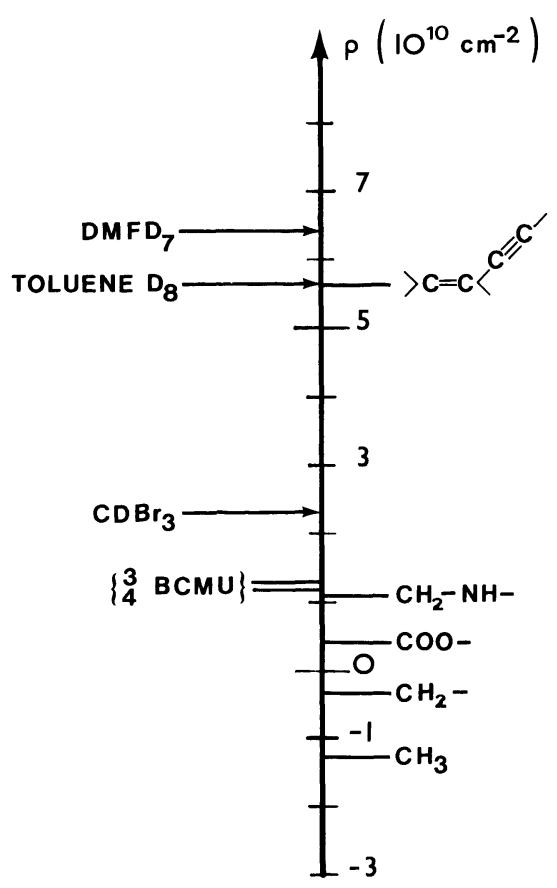

Fig. 1. - Neutron scattering length densities for P-3 and 4BCMU and the solvents used in this study, at room temperature. On the left hand side, monomers are taken as elementary scatterers. On the right hand side, the backbone and various parts of side groups are considered as elementary scatterers. solvents [24], required to normalize the data, were then calculated by taking monomers as elementary scatterers. The polymer molar volumes were assumed to be temperature independent. The temperature variation of the toluene molar volume, which causes a contrast factor variation, was calculated assuming the same thermal expansion coefficient as for benzene $\alpha=12.23 \times 10^{-4} \mathrm{~K}^{-1}$ [25]. That of $\mathrm{N}, \mathrm{N}$-dimethylformamid was calculated using the same thermal expansion coefficient as for $\mathrm{N}$ methylformamid : $\alpha=8.69 \times 10^{-4} \mathrm{~K}^{-1}$ [26]. The influence of added triethylamine was calculated, and found negligible. Finally, one should remember that neutron contrast factors for nuclear scattering do not depend on the electronic configuration of the scattering elements.

2.3 LIGHT SCATTERING EXPERIMENTS. - Light scattering experiments were performed at room temperature. Red solutions of the high molecular weight P-4BCMU in toluene were studied at various concentrations between $0.47 \times 10^{-3} \mathrm{~g}^{-1}$ and $3.7 \times$ $10^{-3} \mathrm{~g} \mathrm{l}^{-1}$.

Static and dynamic light scattering was recorded simultaneously as described earlier [6, 27]. The light source was a $\mathrm{Kr}$-ion laser operating at $6471 \AA$, far beyond the absorption edge of P-4BCMU in the red phase. A few control measurements of the scattering intensities were also performed at $6764 \AA$, that always confirmed the $6471 \AA$ results.

Dust particles were removed by centrifugation in a Beckman L5 centrifuge with the SW 25.1 swinging bucket rotor. The centrifugation time was usually 1 hour at 7000 r.p.m. corresponding to $5000 \mathrm{~g}$. After this procedure, the concentrations were checked by optical absorption. Except for the two lowest concentrations, they decreased by up to a factor of two. The refractive index increment was $\mathrm{d} n / \mathrm{d} c=0.24 \mathrm{ml}$ $\mathrm{g}^{-1}$ as reported earlier [16].

\section{Neutron scattering from P-3BCMU and P- 4BCMU solutions in good solvents.}

The conformational statistics of chain molecules can be studied by radiation scattering techniques [28, 29]. The aim is to obtain the structure factor of one single macromolecule $S(\mathbf{q})$, or its isotropic average $S(q)$ if chain orientations are random, as is the case in the present study. $S(\mathbf{q})$ is just the Fourier transform of the autocorrelation function of the monomer density $\langle\rho(\mathbf{r}) \rho(0)\rangle$. This goal can be reached by use of the Zimm extrapolation method [30]. Then, depending on the spatial resolution, two distinct approaches can be used :

(i) in the Guinier range, i.e. at small $q$ values : for homopolymers, $S(q)$ yields the mean square radius of gyration of the chain $R_{\mathrm{G}}^{2}$. The conformational statistics is then studied by determination of $R_{\mathrm{G}}^{2}$ as a function of the molecular weight $M$. This requires 
well characterized samples in a broad range of $M$ values ;

(ii) at higher $q$ values, i.e. for $q R_{\mathrm{G}} \gg 1: S(q)$ reflects directly intrachain correlations. Informations about the average chain conformation are then obtained from a single polymer sample and polydispersity can be neglected. The traditional route is to compare experimental $S(q)$ functions with predicted ones, calculated from realistic models.

Here, neutron scattering experiments from solutions of high molecular weight P-3 and 4BCMU in good solvents are reported. Since we are concerned with the second approach, before presenting and discussing the results, we survey the models that are expected to describe the internal structure of these polymers.

3.1 MODELS FOR THE CHAIN STRUCTURE FACTOR. Following the first approach, light scattering experiments have been performed from dilute solutions of P-TS12 in 1,2 dichloroethane, a good solvent [6]. The results, corrected for polydispersity, indicated a coil structure and were explained by the PorodKratky wormlike chain model [31]. In this model, a macromolecule is represented by a curve in three dimensional space which shows a continuous transition from rod to coil by increasing its contour length $L$. The relevant parameter is the persistence length $b / 2$, allowing one to distinguish between rigid and flexible behaviors through the ratio $L / b$. It is defined by

$$
\left\langle\dot{\mathbf{r}}\left(s_{1}\right) \dot{\mathbf{r}}\left(s_{2}\right)\right\rangle=\exp \left\{-\frac{2\left(s_{1}-s_{2}\right)}{b}\right\}
$$

where $s_{i}$ is the curvilinear coordinate of an arbitrary point $i$ along the curve; and $\mathbf{r}\left(s_{i}\right)$, the vector indicating the position of this point in three dimensional space. Thus, $\dot{\mathbf{r}}\left(s_{i}\right)=(\mathrm{dr} / \mathrm{d} s)_{i}$ is the unit vector characterizing the direction of the tangent to the curve at point $i$. In the harmonic approximation, the persistence length is proportional to the inverse of absolute temperature [32]. In the limit of the infinitely long chain, it is also half the Kuhn statistical length [33]. For P-TS12 in 1,2 dichloroethane at room temperature, it has been found to be $190 \AA$ from light scattering experiments and $150 \AA$ from viscosity measurements [6].

We can argue that P-3 and 4BCMU in good solvents should behave as wormlike chains. Indeed, the conjugated structure of their backbone is presumably the main cause of stiffness for these polymers and is identical with that of P-TS12. Following the second approach mentioned above, we can therefore expect to observe the form factors of the Porod-Kratky model. More precisely, the $q^{-1}$ scattering behavior of a rod like molecule should be measured since the form factor $P(q)=S(q) / S(0)$ of an infinitely long wormlike chain has the asymptotic form [34] :

$$
\lim _{\substack{L \rightarrow \infty \\ q \rightarrow \infty}} \frac{L}{b} P(q)=\frac{\pi}{q b}+\frac{4}{3(q b)^{2}}
$$

and, for long enough chains $(L / b>2)$, equation (4) holds for $q b>7$ as shown in figure 2 .

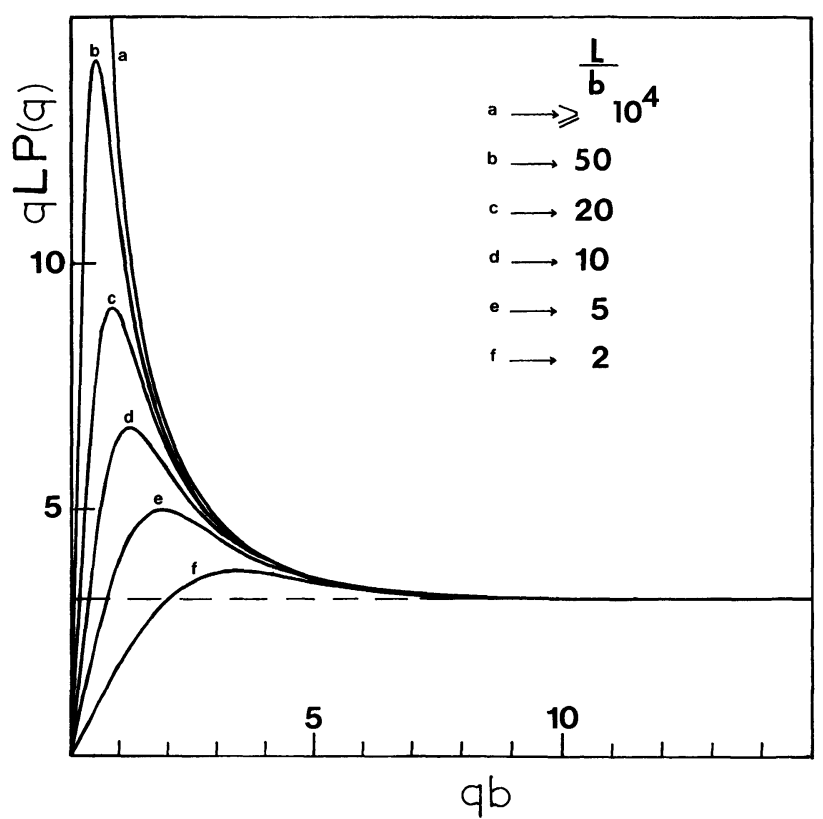

Fig. 2. - Chain form factors according to the PorodKratky model, in a plot of $q L P(q)$ versus $q b$. The characteristic $q^{-1}$ scattering behavior is observed for $q b>7$ as $L / b>2$.

When the spatial resolution is increased, this model is no longer accurate because macromolecules can no longer be approximated by thin threads [35]. The size of the large side groups of P-3 and 4BCMU is no longer negligible and the $q^{-1}$ decay predicted by equation (4) might be obscured. Depending on the spatial scale, this effect of the side groups can be treated as a simple thickness. The chain structure factor is then written as $[36,37]$ :

$$
S(q)=S_{0}(q) \Phi(q)
$$

where $S_{0}(q)$ is the form factor of the curved line previously associated to the polymer and $\Phi(q)$, that of its cross section. Such a decomposition assumes the conformation of side groups to be independent of the position along the main chain and of the structure of the backbone. This implies that the distribution of hydrogen bonds, if any is present [ 1 , 38 ], is the same all along the chain when averaged over a distance comparable to the maximum spatial resolution of the experiment, of the order of $40 \AA$. Indeed, no long range cooperativity among hydrogen bonds is expected, unlike in polypeptides. The simplest way is then to use the Guinier approxi- 
mation for $\Phi(q)$, and equation (5) is replaced by [39] :

$$
S(q)=S_{0}(q) \exp \left\{-\frac{q^{2} R_{\mathrm{c}}^{2}}{2}\right\}
$$

$R_{\mathrm{c}}^{2}$ being the mean square radius of gyration of the cross section where the role of masses is played by scattering length densities. This exponential term requires $q R_{\mathrm{c}}<1$ and, depending on the $R_{\mathrm{c}}^{2}$ value, can change drastically the scattering behavior of the wormlike chain.

Alternatively, if side groups can be considered as quasi homogeneous chains roughly localized in a plane, the cross section term should lead to the characteristic $q^{-2}$ scattering behavior of a flat particle. Indeed the form factor of a stiff or slightly bending ribbon with surface $S$ is [36, 39]:

$$
P(q)=\frac{2 \pi}{S q^{2}}
$$

for $1 / W \ll q<1 / T$, where $W$ and $T$ are the width and the thickness of the ribbon, respectively [40].

Finally, a model of misoriented rigid platelets of variable length can also be considered [4, 7, 8]. Rotations around $\sigma$ bonds are then involved to break the conjugation, the planar structure being lost locally. Obviously, the cross section term should be the same as the one for the continuous model. In particular, the $q^{-2}$ decay may be observed. However, we can argue that the form factor $S_{0}(q)$ should differ somewhat from that of a continuously curved line if rigid units are long enough. As explained in the appendix, it does indeed show the characteristic $q^{-1}$ decay but its asymptotic form does not correspond exactly to equation (4) as the compactness of the structure is slightly different. The statistical zigzag model, yields :

$$
\lim _{\substack{L \rightarrow \infty \\ q \rightarrow \infty}} \frac{L}{b} P(q)=\frac{\pi}{q b}+\frac{\pi^{2}-4}{(q b)^{2}}
$$

where $b / 2$ is now the average length of rods. Remarkably, for long enough chains, equation (8) still holds for $q b>7$.

\subsection{RESUl.TS.}

3.2.1 P-3BCMU. - The structure factor of a single P-3BCMU-1 chain in perdeuteriated DMF has been obtained from the scattering of four dilute solutions, applying the Zimm extrapolation procedure. The experiments were performed at room temperature and the dimensionless form factor $g(q)$, defined as :

$$
g(q)=\frac{M_{\mathrm{w}}}{m} P(q)
$$

where $m$ is the molar mass of monomers, is shown in

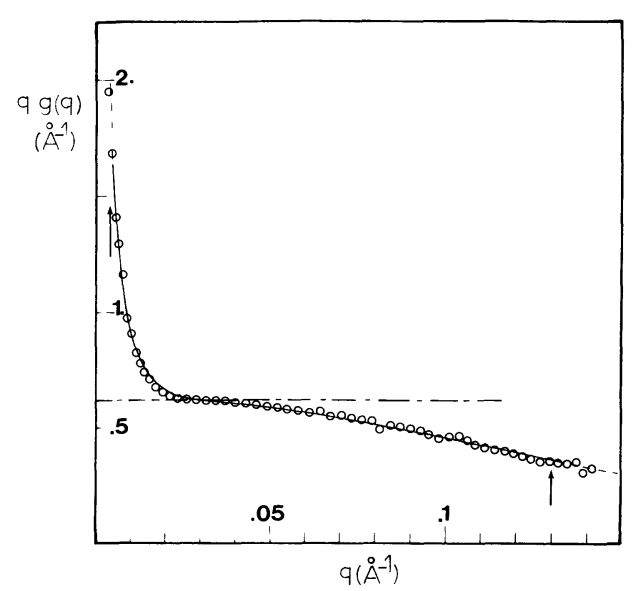

Fig. 3. - Holtzer plot of the form factor of one P-3BCMU-1 chain in perdeuteriated DMF, at room temperature. Circles are experimental data and the full line represents the best fit by the theoretical structure factor $g(q)=g_{0}(q) \exp \left(-q^{2} R_{\mathrm{c}}^{2} / 2\right)$. The wormlike chain model is assumed for $g_{0}(q)$ and arrows indicate the used $q$-range. The dashed line corresponds to the height of the $q^{-1}$ plateau which yields the mass per unit contour length $M_{\mathrm{L}}$.

figure 3, using the Holtzer representation [41]. It is in agreement with a rigid coil structure.

At small $q$ values, $g(q)$ is indeed proportional to $q^{-1}$ in the range $0.023<q<0.04 \AA^{-1}$, corresponding to a statistical length $b$ close to $300 \AA$. According to equations (9) and (4) (or (8)), the mass per unit contour length $M_{\mathrm{L}}=M / L$ can be deduced from the height $\mathcal{A}$ of the $q^{-1}$ plateau appearing in figure 3 . $\mathcal{A}=\pi M_{\mathrm{L}} / m$ so $M_{\mathrm{L}}=94 \mathrm{~g}$ mole $^{-1} \AA^{-1}$, i.e. a contour length per monomer unit of the order of $5 \AA$. This is the expected value for a P-3BCMU chain in the trans configuration of the double bonds, whereas a chain in the cis configuration of the double bonds would have $M_{\mathrm{L}}($ cis $) \simeq$ $1.4 M_{\mathrm{L}}($ trans $)=132 \mathrm{gmole}^{-1} \AA^{-1}$. The experimental uncertainty is of the order of $8 \mathrm{~g} \mathrm{~mole} \mathrm{~m}^{-1}$ $\AA^{-1}$, so even taking into account the uncertainty in calculating the polymer molar volume involved in the contrast factor, the cis value is ruled out. This shows that P-3BCMU chains in DMF are, on the average, in the trans configuration of the double bonds. Since their visible absorption spectrum is typical of all yellow polydiacetylene solutions, it may be already expected that this is the usual configuration of these polymers in a good solvent. Now, using the Kratky representation for the data, we may attempt to choose between the wormlike and discrete defect chain models. As discussed in 3.1 and appendix, the main properties of $g(q)$ are fixed by $b$ and $M_{\mathrm{L}}$, leading to asymptotes of the same slope $\pi M_{\mathrm{L}} / m$ in a plot of $q^{2} g(q)$ versus $q$. However, the points where these asymptotes cross the $q^{2} g(q)$ axis depend on the model. The corresponding values are 


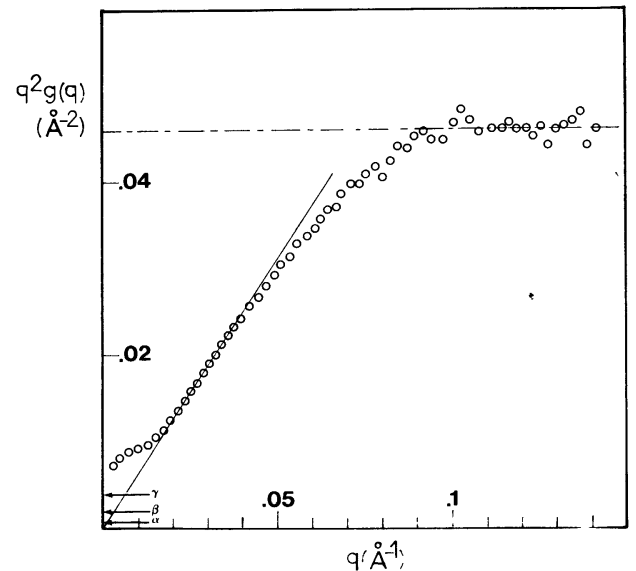

Fig. 4. - Kratky plot of the form factor of one P-3BCMU-1 chain in perdeuteriated DMF, at room temperature. Circles are experimental data. The straight line through the origin is the $q^{-1}$ asymptote. Its slope is fixed by the $M_{\mathrm{L}}$ value obtained from the height of the $q^{-1}$ plateau in figure $3\left(94 \mathrm{~g} \mathrm{~mole}^{-1} \AA^{-1}\right) . \alpha$ is the cross point between this asymptote and the $y$-axis predicted by the wormlike chain model for $b=310 \AA$. $\beta$ and $\gamma$ are those corresponding to the regular and statistical zig zag models, respectively. The dashed line represents the height of the $q^{-2}$ plateau which yields the mass per unit area $M_{\mathrm{S}}$.

given in the appendix and indicated in figure 4 as : $\alpha$, for the wormlike chain; $\beta$ and $\gamma$, for chains formed by regular and statistical rigid segments, respectively. Only the first agrees with the experiment, indicating that rigid elements must be short so the number of defects must be high. According to the relation $b / 2=\ell(1-\cos \phi)^{-1}$ [31], the angle $\phi$ between successive rigid units of length $\ell$ should also be small in order to explain the measured $b$ value. Therefore, the continuous wormlike chain model is the best approximation, at least for $q<0.04 \AA^{-1}$, beyond which the side-groups extension becomes significant. In other words, the internal structure of these coils is less compact than that of a broken chain with a small number of local defects.

At higher $q$ values, information about the average conformation of the side groups can be obtained. As shown in figure 3 , the $q^{-1}$ scattering behavior is no longer observed for $q>0.04 \AA^{-1}$; this is due to the effect of the chain cross section. Assuming the wormlike chain model for $g_{0}(q)$, equation (6) was used to fit the data in the range of scattering vectors : $0.005<q<0.13 \AA^{-1}$. Four parameters are involved: $b$ and $M_{\mathrm{L}}$, which are approximately known, as shown previously; the chain contour length $L$ and the mean square radial radius of gyration $R_{\mathrm{c}}^{2}$. Note that $R_{\mathrm{c}}^{2}$ cannot be obtained directly from a plot of $\log (q g(q))$, versus $q^{2}$, although such data representation allows a rough test of the exponential approximation, equation (6). It was first checked that $L$ plays no role in this $q$ range, showing that polydispersity can be neglected. The set of parameters $\left(b, M_{\mathrm{L}}, R_{\mathrm{c}}^{2}\right)$ which gives the best fit of the experimental form factor is given in table III, and the corresponding curve is presented in figure 3. Remarkably $R_{\mathrm{c}}^{2}$ is found to be smaller than the value expected for fully extended side groups, suggesting some disorder. On the other hand, as shown in figure $4, g(q)$ scales like $q^{-2}$ for $0.1<q<0.15 \AA^{-1}$. This scattering behavior can be ascribed to a ribbon like structure, since a side group can be considered as a quasi-homogeneous particle at the spatial reolution of the experiment $2 \pi / q_{\max } \sim 40 \AA$. Equation (7) applies up to $q=0.14 \AA^{-1}$ at least, so the ribbon thickness is smaller than $7 \AA$, and the height $\mathcal{B}$ of the $q^{-2}$ plateau appearing in figure 4 provides the mass per unit area $M_{\mathrm{S}}=M / S$, where $S$ is the ribbon surface. According to equations (9) and (7), $\mathfrak{B}=2 \pi M_{\mathrm{S}} / m$ and we find $M_{\mathrm{S}}=3.50 \pm 0.15 \mathrm{~g} \mathrm{~mole}^{-1} \AA^{-2}$. From the $M_{\mathrm{L}}$ value obtained previously, the effective width of the ribbon $\tilde{\omega}$ is : $\tilde{\omega}=M_{\mathrm{L}} / M_{\mathrm{S}}=27 \pm 1.5 \AA$. Fully extended side groups would correspond to a width of the order of $35 \AA$ for P-3BCMU. These values cannot be precisely compared at present because the side -groups are not densely packed along the ribbon, the distance between the points where they are attached to the chain being $\sim 5 \AA$. However, this result suggests that side groups are gradually disordering from their inner end bound to the conjugated backbone, out to

Table III. - Structural parameters of P-3 and 4BCMU chains in good solvents.

\begin{tabular}{|c|c|c|c|c|c|c|}
\hline Sample & Solvent & $\begin{array}{c}T \\
(\mathrm{~K})\end{array}$ & $\begin{array}{c}b \\
(\AA)\end{array}$ & $\begin{array}{c}M_{L} \\
\left(\mathrm{~g} \mathrm{~mole}^{-1} \AA^{-1}\right)\end{array}$ & $\begin{array}{c}R_{\mathrm{c}}^{2} \\
\left(\AA^{2}\right)\end{array}$ & $\begin{array}{c}\tilde{\omega} \\
(\AA)\end{array}$ \\
\hline P-3BCMU-1 & $\begin{array}{l}\text { DMFD }_{7} \\
\text { DMFD }_{7}\end{array}$ & $\begin{array}{l}295 \\
333\end{array}$ & $\begin{array}{l}310 \pm 6 \\
280 \pm 15\end{array}$ & $\begin{array}{l}94.3 \pm 1.0 \\
94 \pm 3\end{array}$ & $\begin{array}{l}63.7 \pm 3.0 \\
64 \pm 6\end{array}$ & $27 \pm 1.5$ \\
\hline P-4BCMU-1 & $\begin{array}{l}\text { Toluene } \mathrm{D}_{8} \\
\text { DMFD }_{7}\end{array}$ & $\begin{array}{l}343 \\
333\end{array}$ & $\begin{array}{l}278 \pm 10 \\
285 \pm 15\end{array}$ & $\begin{array}{c}96.9 \pm 0.5 \\
104 \quad \pm 5\end{array}$ & $\begin{array}{l}60.2 \pm 1.0 \\
59 \pm 3\end{array}$ & $25 \pm 1$ \\
\hline
\end{tabular}


their terminal methyl group. More precisely, the inner region would be fairly well ribbon like, and the outer end more randomly oriented. Increasing the chemical resolution, using selectively deuteriated polymers, and the spatial resolution, in order to look for the specific oscillations of the form factor of a ribbon [40], would yield a more accurate description of side group conformations.

Similar results have been obtained at $T=60^{\circ} \mathrm{C}$. Within the experimental uncertainty, the persistence length is in agreement with the $T^{-1}$ law [32], while $R_{\mathrm{c}}^{2}$ remains constant, as indicated in table III.

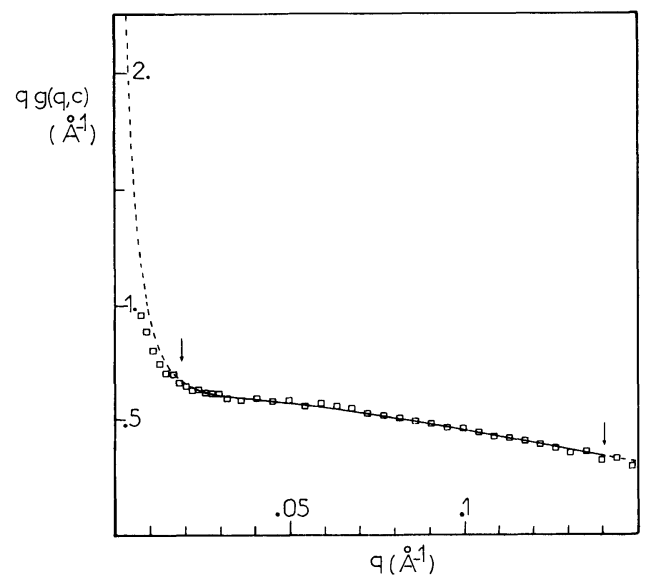

Fig. 5. - Holtzer plot of the scattering curve $g(q, c)$ for P$4 \mathrm{BCMU}-1$ in perdeuteriated toluene at $T=70^{\circ} \mathrm{C}$ for $c=4.3 \mathrm{gl}^{-1}$. Intermolecular correlations are negligible for $q>0.02 \AA^{-1}$ and the full line represents the best fit by the theoretical structure factor $g(q)=g_{0}(q) \exp \left(-q^{2}\right.$ $\left.R_{\mathrm{c}}^{2} / 2\right)$. The wormlike chain model is assumed for $g_{0}(q)$ and arrows indicate the used $q$-range.

3.2.2 P-4BCMU. - P-4BCMU-1 solutions in perdeuteriated toluene are yellow for $T>66^{\circ} \mathrm{C}$. The scattering behavior for a concentration $c=4.3 \mathrm{~g} \mathrm{l}^{-1}$ has been studied as a function of temperature as described in Section 4. At $T=70^{\circ} \mathrm{C}$, the result is given in figure 5 , using the dimensionless structure factor $g(q, c)$ defined as :

$$
g(q, c)=\frac{m}{K^{2} c \mathcal{N}} \Sigma^{\mathrm{coh}}(q)
$$

where $c\left(\mathrm{~g} \mathrm{~cm}^{-3}\right)$ is the polymer concentration and $K^{2}\left(\mathrm{~cm}^{2}\right)$, the neutron contrast factor. Comparison with the scattering from another solution with $c=1.2 \mathrm{~g} \mathrm{l}^{-1}$, shows that intermolecular correlations are negligible beyond $q=0.02 \AA^{-1}$. Therefore, in the corresponding $q$-range, $g(q, c)$ is identical to $g(q)$ defined by equation (9). Obviously this scattering behavior is similar to that of $\mathrm{P}-3 \mathrm{BCMU}$ in perdeuteriated DMF and the same analysis can be carried out. Thus, assuming the wormlike chain model for the scattering function of the thin thread associated to the macromolecule and using the exponential form (6) we have determined the set of parameters $\left(b, M_{\mathrm{L}}, R_{\mathrm{c}}^{2}\right)$ which reproduces in the best way the experimental scattering curve in the $q$ range : $0.02<q<0.14 \AA^{-1}$. The results are given in table III and the corresponding form factor is shown in figure 5. Correcting for the temperature dependence of the persistence length, yields $b=323 \AA$ at room temperature. This value is close to that obtained for P-3BCMU. Again $M_{\mathrm{L}}$ is in agreement with a trans isomeric configuration of the double bonds. Moreover, this remains true to within half a degree from the onset of the yellow $\rightarrow$ red color transition studied in section 4 . Therefore, the model which relates this transition to the occurrence on cooling of a cis $\rightarrow$ trans isomerization [9], cannot be correct in this case. The average side groups conformation is also found to be close to that of P-3BCMU in $\mathrm{DMFD}_{7}$. Yet we note that $R_{\mathrm{c}}^{2}$ is slightly smaller, although P-4BCMU side groups have one extra $\mathrm{CH}_{2}$. Such a small difference in the $R_{\mathrm{c}}^{2}$ values may not be apparent by mere comparison of figures 3 and 5 , but it is immediately seen on plots of Log $[q g(q)]$ versus $q^{2}$, a comparison which is meaningful since the persistence lengths are equal. Similarly, the effective width of the ribbon like structure $\tilde{\boldsymbol{\omega}}$, is also smaller for P-4BCMU (Tab. III). This is not a temperature effect and can explain that $\mathrm{P}-4 \mathrm{BCMU}$ is more soluble than $\mathrm{P}-3 \mathrm{BCMU}$ in the same solvent. Similarly, it was found that P-4BCMU solutions and $\mathrm{P}-3 \mathrm{BCMU}$ solutions in $\mathrm{DMFD}_{7}$ at $T=60^{\circ} \mathrm{C}$ yield nearly identical worm-like chain parameters, but $R_{\mathrm{c}}^{2}$ for $\mathrm{P}-4 \mathrm{BCMU}$ is slightly smaller than for $\mathrm{P}$ 3BCMU (Tab. III). This difference may be related to the observation, made on molecular models, that hydrogen bonds are more easily formed in $\mathrm{P}$ 3BCMU without strain on the conjugated backbone. However no clear conclusion is possible, since the bonds formed in solution have a lower energy than in the solid, and presumably a different geometry. Once again, note that $R_{\mathrm{c}}^{2}$ is not sensitive to temperature between 20 and $70{ }^{\circ} \mathrm{C}$.

We also would like to mention some results obtained for $\mathrm{P}-4 \mathrm{BCMU}$ yellow solutions in $\mathrm{CDBr}_{3}$. As shown in figure 1 , the corresponding contrast situation is much less favorable, so less accurate data were obtained. It could be shown however that in $\mathrm{CDBr}_{3}$ as well, this polymer form a semi-rigid chain, but with a larger statistical lengths $(b=410 \pm 30 \AA$ at room temperature). Accurate determination of $M_{\mathrm{L}}$ is no longer straightforward, since preferential adsorption onto the polymer of ethanol molecules used as solvent stabilizers is possible. Further data on this system are presented and discussed elsewhere $[38,42]$.

3.3 Discussion. - P-3BCMU and P-4BCMU chains in the corresponding good solvents have 
similar internal structures. They can be described by the wormlike chain model at spatial scales related to distances larger than $150 \AA$, and by a wormlike ribbon model for distances larger than $40 \AA$.

The interrupted rod model, proposed to rationalize the optical density measurements $[4,7$, 9], is not adequate at least in its initial form. There may be a large number of weakly disorientational defects, rather than a small number of large disturbances. Thus the delocalized $\pi$ electrons experience a large number of small potential fluctuations, not a small number of large ones. This may have consequences in the calculation of the electronic properties of such chains.

In all yellow solutions, P-3 and 4BCMU are in the trans configuration of the double bonds. The same is likely to be true for P-TS12 and possibly for all polydiacetylenes dissolved in good solvents which all have very similar visible absorption spectra. This result rules out a cis $\rightarrow$ trans isomerization as the explanation of the yellow $\rightarrow$ blue (or red) color transition [9].

The persistence lengths $b / 2$ of P-3 and 4BCMU in good solvents are close to $160 \AA$ at room temperature and the data are in agreement with a $T^{-1}$ dependence for $b$. Using experimental force constants, Allegra et al. have calculated a persistence length of the order of $170-190 \AA$ at room temperature due only to harmonic thermal motions around bonds, for a perfect all-trans polydiacetylene chain without side-groups [43]. The same value of $b$ has been obtained by viscosity measurements for $P$ TS12, a polydiacetylene without hydrogen bonds between adjacent side groups [6]. All this suggests that the rigidity of polydiacetylenes in good solvents is mainly caused by the conjugated structure of their backbone; thus hydrogen bonds, whether or not some of them are formed, do not contribute significantly to the backbone rigidity of P-3 and 4BCMU chains in good solvents, so equation (5) is indeed a valid approximation.

The average side group conformation is slightly more extended for P-3 than for P-4BCMU in good solvents. For both polymers, it does not correspond to full extension. Most likely, disorder of the side groups increases from the carbon chain backbone to the terminal methyl group. A more detailed study would require experiments at larger spatial resolution and with selectively labeled side groups.

\section{Neutron scattering study of the color transition for P-4BCMU in toluene.}

For a structural study of the color transition, P4BCMU in toluene seems to be a more suitable system than P-3BCMU or P-4BCMU in chloroformhexane mixtures in which preferential adsorption phenomena cannot be excluded, and are difficult to take into account quantitatively. For a given molecular weight distribution, the only parameter governing the solvent quality is then temperature : yellow solutions of P-4BCMU in toluene turn red when cooled from high temperatures $[5,6]$. This color transition corresponds to changes in the electronic structure of the conjugated backbone of the macromolecules. The related structural modifications are not well known [12-14, 16-17]. For high $M_{\mathrm{w}}$ P4BCMU samples and polymer concentrations larger than a critical value $c_{\mathrm{G}}$, gel formation occurs. It has been proposed that $c_{\mathrm{G}}=0.6 \mathrm{gl}^{-1}[13,14]$, but $c_{\mathrm{G}}$ should be related to an overlap of the polymers and therefore should depend on the average molecular weight $M_{\mathrm{w}}$ and polydispersity, in a way which has not been investigated yet. Moreover, for polymer concentrations less than $0.01 \mathrm{gl}^{-1}$ light scattering experiments have been interpreted as evidence of red solutions of isolated macromolecules, suggesting that the color transition could be a pure intramolecular coil to rod modification [13]. We return to this problem of the existence of true red solutions of isolated rod molecules in the next section. We report here neutron scattering experiments performed at different temperatures between $80^{\circ} \mathrm{C}$ and $38^{\circ} \mathrm{C}$, on

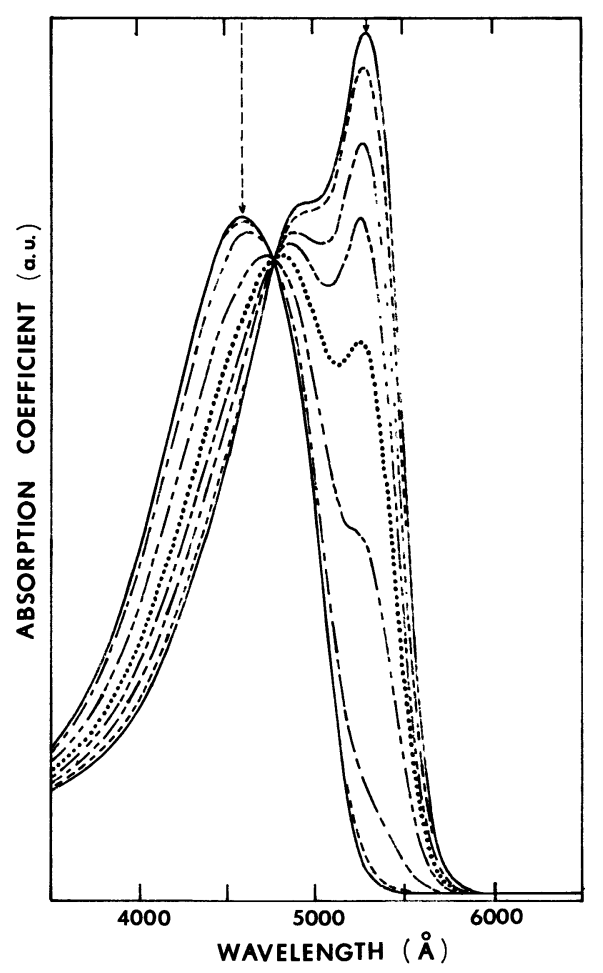

Fig. 6. - Absorption spectra for P-4BCMU-1 in toluene at different temperatures : $67.2 ; 66.1 ; 65.0 ; 63.9 ; 62.9$; $61.8 ; 60.7 ; 58.6$ and $57.5^{\circ} \mathrm{C}$. The solid curve peaking at $4600 \AA$ (dashed arrow) corresponds to the highest temperature and the solid curve peaking at $5300 \AA$ (solid arrow) to the lowest temperature. There is an isosbestic point suggesting a coexistence of two electronic states. 
cooling a high molecular weight P-4BCMU solution in perdeuteriated toluene.

It was first checked that solvent deuteriation does not influence the transition: protonated and perdeuteriated toluene solutions yield identical visible and IR absorption spectra, as well as identical D.S.C. results [44]. The visible absorption spectra of dilute toluene solutions of the same P-4BCMU as used in the neutron scattering experiments, and referred to as P-4BCMU-1, are shown in figure 6 .

The transitional region, for which curves can be described by fractional superposition of the two limiting spectra corresponding to the red and yellow phases is found in the range $58<T<66^{\circ} \mathrm{C}$. A slightly lower transition onset temperature and a much more gradual color change have been reported in the literature [13]. Such differences demonstrate the importance of a good characterization of the samples. They are presumably due to varying fractions of shorter P-4BCMU chains, a larger polydispersity leading to a broader transitional temperature range, although different preparations of these polymers may also lead to different concentrations of defects in the backbone or in the side groups. On the other hand, the transition occurs upon cooling at the same temperature of $66^{\circ} \mathrm{C}$ in a wide range of polymer concentrations from the smallest concentration accessible to spectroscopic study up to $c=20 \mathrm{gl}^{-1}$, studied in D.S.C. experiments [44]. The transition shows a large hysteresis and all results presented in this section were obtained by decreasing the temperature. The polymer concentration was $c=4.3 \mathrm{gl}^{-1}$. A stable gel is formed, showing no detectable syneresis at room temperature for up to six months at least.

The system may not be in thermodynamic equilibrium when studied at a temperature below the onset of the color transition. It is known that the absorption spectrum of P-3BCMU in chloroform-hexane mixtures at room temperature near the critical concentration is time dependent even on long time scales $[11,45]$. In the present work, kinetic effects were observed on the neutron scattering function, that is, on the structure of the system; however, these changes were negligible on the time scale of $10^{3} \mathrm{~s}$, a typical duration of the measurement at a given temperature. On the time scale of the hour, changes were observed, but they were still smaller than those due to a temperature decrease of $1^{\circ} \mathrm{C}$. These kinetic effects were not investigated in detail, and they could be neglected thanks to the high neutron flux at I.L.L. allowing short duration experiments. On the same time scale, the visible absorption spectra did not change perceptibly : either the kinetic effects are sensitive to polymer concentration (which was smaller for the absorption measurements) or they only affect the structure and not the electronic properties. Therefore, both scattering and absorption experiments were performed in a quasistationary state, and it is meaningful to compare them.

Since intermolecular correlations cannot be neglected a priori, results will be discussed in terms of the coherent differential cross section $\Sigma^{\text {coh }}(q)$ and/or the dimensionless scattering function $g(q, c)$ defined by equation (10).

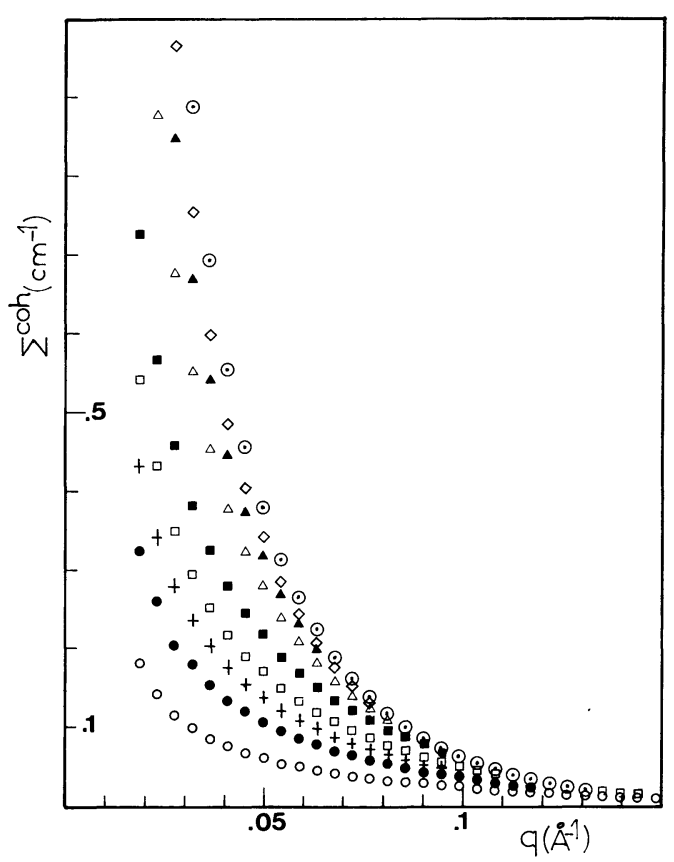

Fig. 7. - Coherent differential cross sections $\Sigma^{\text {coh }}(q)$ of a $c=4.3 \mathrm{gl}^{-1} \mathrm{P}-4 \mathrm{BCMU}-1$ solution in perdeuteriated toluene at different temperatures : $T=70{ }^{\circ} \mathrm{C}(\mathrm{O}), 65.1^{\circ} \mathrm{C}$ $(\bullet), 63.8^{\circ} \mathrm{C}(+), 62.8^{\circ} \mathrm{C}(\square), 60.8^{\circ} \mathrm{C}(\square), 58^{\circ} \mathrm{C}(\triangle)$, $55.4^{\circ} \mathrm{C}(\Delta), 50.6{ }^{\circ} \mathrm{C}(\diamond)$, and $37.8^{\circ} \mathrm{C}(\odot)$. Between $80^{\circ} \mathrm{C}$ and $67^{\circ} \mathrm{C}$ the solution is yellow and the results are nearly identical. Therefore only the curve corresponding to $70^{\circ} \mathrm{C}$ is presented.

As shown in figure 7, the color transition is related to a major modification of the structure of the solution. For $T>66^{\circ} \mathrm{C}$, in the yellow phase $\Sigma^{\text {coh }}(q)$ does not vary with $T$, except for small differences related to the temperature dependence of the contrast factor. At $T=66{ }^{\circ} \mathrm{C}$, the solution becomes red and a fast increase of $\Sigma^{\mathrm{coh}}(q)$ is observed in the whole investigated $q$-range. At small $q$ values (less than about $\left.0.07 \AA^{-1}\right), \Sigma^{\text {coh }}(q)$ keeps increasing as the temperature decreases, as also observed in light scattering experiments. On the other hand, at higher $q$ values, $\Sigma^{\text {coh }}(q)$ levels off at $T \sim 58^{\circ} \mathrm{C}$ and even decreases at lower temperatures, as shown in figure 8. Since the optical transition range is $66>T>58^{\circ} \mathrm{C}$, this suggests that two kinds of structural modifications occur, above and below $58{ }^{\circ} \mathrm{C}$ respectively.

The change in the scattering behavior of the solution below $66^{\circ} \mathrm{C}$ cannot be due to an in- 


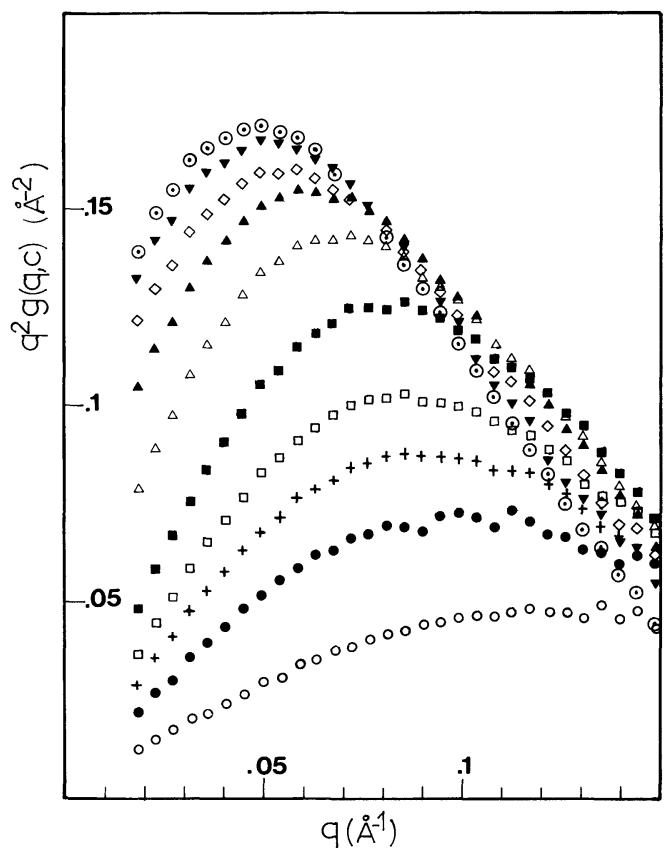

Fig. 8. - Kratky plot of the scattering curve $g(q, c)$ corresponding to the coherent differential cross sections given in figure 7 . The scattering curve for the additional temperature $T=45^{\circ} \mathrm{C}(\nabla)$ is also reported.

tramolecular modification alone, in which case the mass per unit contour length could change by a small amount only. Furthermore, the optical absorption redshift suggests an increase of the chain rigidity and therefore a larger persistence length. $\Sigma^{\text {coh }}(q)$ should then decrease near the small $q$ end of the $q^{-1}$ plateau that appears in figure 3 ; this is not observed. Thus the variation of $\Sigma^{\text {coh }}(q)$ or $g(q, c)$ reported in figures 7 and 8 can only correspond to a sudden occurrence of aggregation at the color transition. This is not really surprising at the concentration studied, but one may emphasize that the internal structure of the macromolecules in the yellow solution is not modified down to about one half-degree above the onset of the transition. No precursor intramolecular change for the color transition is observed, neither in the stiffness of the thin thread nor in the average conformation of side groups.

Neglecting at first intermolecular correlations, two assumptions can be made to infer structural information from the dimensionless function $g(q)$. Either the system contains, at all temperatures, a single kind of scattering objects ; then the variations of $g(q)$ with $T$ correspond to the temperature dependence of the internal structure of these objects. Or the system, at least between 66 and $58^{\circ} \mathrm{C}$, contains two or more types of objects; $g(q)$ is then the sum of several correlation functions, which should be separated in order trying to extract information about the internal structure of the scatterers. From the present scattering data alone, we cannot decide between the two points of view. There is, however, some evidence in favor of the second one.

As mentioned already in other papers [4, 13], in the transitional range, the visible absorption spectra of dilute P-4BCMU solutions in toluene obey quite accurately the relation

$$
\varepsilon(T, \lambda)=[1-X(T)] \varepsilon_{\mathrm{y}}(\lambda)+X(T) \varepsilon_{\mathrm{r}}(\lambda)
$$

where $\varepsilon_{\mathrm{y}}(\lambda)$ and $\varepsilon_{\mathrm{r}}(\lambda)$ are limiting absorption curves for purely yellow and purely red phases respectively and $X(T)$ a temperature-dependent fraction of monomers in the red state. Since $\varepsilon_{\mathrm{y}}(\lambda)$ at least is slightly temperature dependent [38], and the same may be true for $\varepsilon_{\mathrm{r}}(\lambda)$, a relation like (11) has meaning only when the transitional range is narrow, as is the case here, and if the reference spectra $\varepsilon_{\mathrm{y}}(\lambda)$ and $\varepsilon_{\mathrm{r}}(\lambda)$ are taken at temperatures respectively just above and just below the transitional range. In the present case one may choose 67 and $57{ }^{\circ} \mathrm{C}$, since in dilute solutions of the high molecular weight $\mathrm{P}-4 \mathrm{BCMU}$ used in this study, $X(T)=0$ for $T>66^{\circ} \mathrm{C}$ and $X(T)=1$ for $T<58^{\circ} \mathrm{C}$. The function $X(T)$ is not the same for all $\mathrm{P}-4 \mathrm{BCMU}$ samples. It depends on the average molecular weight (see 5.2) and probably also on the polydispersity since, as mentioned above, the samples used in [9, 12-14] have a much broader transitional range. Indeed, the presence of an isosbestic point suggests the coexistence of two electronic states. Note that the concentrations used in the optical measurements may be several orders of magnitude smaller than those used in the neutron scattering experiments, yet the transition temperature onset and range are the same.

It seems reasonable to try and carry out the same decomposition as in equation (11) for the neutron scattering data in the range $66>T>58^{\circ} \mathrm{C}$. Again, one has to consider a red and a yellow component, having the same contrast length (which corresponds to the atomic structure of the monomers) but different structure factors. However, additional assumptions concerning the intermolecular terms are necessary. If upon aggregation the red component segregates from a fluid medium containing the yellow one, the yellow-red cross term is negligible, and a decomposition as in equation (11) is indeed possible. Since the concentration of monomers in the yellow state is certainly less than in the pure yellow solution at higher $T$, intermolecular correlations associated with the yellow component (the yellow-yellow term) will be negligible in the whole $T$-range for $q>0.02 \AA^{-1}$ (cf. $\$ 3.2 . b$ ). Since the yellow component of the visible absorption spectrum is independent of $T$ in the transitional range, it seems reasonable to assume that the corresponding form factor is also independent of $T$ for $q>0.02 \AA^{-1}$. 
Intermolecular contributions to the red structure factor may not be neglected a priori, so the scattering analogue of equation (11) should be written as

$$
\begin{aligned}
& g(q, c, T)=X(T)\left\{g_{\mathrm{r}}(q, c, T)+\right. \\
& \left.\quad+X(T) g_{2 \mathrm{r}}(q, c, T)\right\}+(1-X(T)) g_{\mathrm{y}}(q)
\end{aligned}
$$

where $g_{\mathrm{r}}$ is the form factor of a single red aggregate, $g_{2 r}$ the intermolecular term associated with monomers belonging to different red aggregates, and $g_{\mathrm{y}}$ the temperature-independent form factor of a single yellow chain. $X(T)$ is the same function as in equation (11).

It is indeed possible to fit the present experimental data in the transitional range by an equation similar to (11) that is, with $g_{\mathrm{r}}+X(T) g_{2 r}$ independent of $T$, down to $60{ }^{\circ} \mathrm{C}$. This suggests that, in the $q$-range explored $g_{\mathrm{r}}$ is independent of $T$ between 66 and $60{ }^{\circ} \mathrm{C}$ and that $g_{2 r}$ is negligible, a reasonable result since the interaction between weakly interacting aggregates should be of the excluded volume type and be negligible at spatial scales smaller than the average aggregate size.

A constant $g_{\mathrm{r}}$ indicates constant internal structure of the red aggregates in the $q$-range : $0.02<q<0.15 \AA^{-1}$, corresponding to distances less than a few hundred angströms. The results suggest that their size does not significantly change with $T$, and that the increase of $X(T)$ is due to an increase in the number of aggregates. It might be argued that the very first aggregates have lateral dimensions already larger than a few hundred angströms, corresponding to aggregation numbers of a few hundred at least, in which case aggregate growth upon a decrease of $T$ would not be easily detected here ; however, this would not be easily reconciled with the relatively modest increase in scattering intensity. In all cases, the packing of polymer chains within the aggregates does not change in the transition temperature range.

The $g_{\mathrm{r}}$ obtained using equation (12) is almost identical to the experimental curve at $60.8^{\circ} \mathrm{C}$. It no longer shows a $q^{-1}$ and $q^{-2}$ dependence, suggesting that red aggregates are 3-dimensional objects.

Note that the above treatment does not require that a single polymer chain be entirely either in the yellow or in the red phase, since the spatial scale considered is smaller than both the polymer contour length $L$ and the polymer radius of gyration in the yellow phase. Therefore, this treatment is compatible with the fringed micelles model [17].

As mentioned above, another point of view is to consider that, in the transitional range, the system is not a mixture of two statistically well-defined structures in varying proportions, and contains a single type of object the average geometry of which changes continuously with $T$. The problem of reconciling this point of view with spectroscopic data will not be discussed here. In this model, the following conclusions can be drawn. Above $61^{\circ} \mathrm{C}$, a $q^{-1}$ dependence of $g(q)$ at small $q$ values is still observed, corresponding to a mass per unit length $M_{\mathrm{L}}$ steadily increasing with decreasing $T$, from about $100 \mathrm{~g} \mathrm{~mole}^{-1} \AA^{-1}$ in the yellow phase, to more than $500 \mathrm{~g} \mathrm{~mole}^{-1} \AA^{-1}$ below $61{ }^{\circ} \mathrm{C}$. The disappearance of this $q^{-1}$ dependence shows that the scatterers lose their cylindrical average geometry. This corresponds to modest aggregation numbers. Furthermore, the $q^{-2}$ dependence at large $q$ values disappears below $65{ }^{\circ} \mathrm{C}$, showing that the structures formed are 3dimensional rather than 2-dimensional.

Below $60^{\circ} \mathrm{C}$, the yellow contribution is negligible, and there is no difference between the two above points of view. One is then below the transitional range and DSC shows that a second transformation sets in [44]. The scattering function now changes slowly with $T$. This range will not be discussed further here, but we wish to mention that the aggregates become more and more $3 \mathrm{~d}$ and their compactness increases, as shown by the change of scattering curves at high $q$ values; the characteristic $q^{-4}$ Porod law [39] is observed at $T=55.4^{\circ} \mathrm{C}$ for $q>0.11 \AA^{-1}$. A more detailed study of the transition and of the gel structure is in progress [42].

\section{Search for red solutions of isolated rod molecules.}

It has been suggested that the color transition is a single chain phenomenon corresponding to an intramolecular coil to rod change $[5,11,13]$; aggregation as well as gelation then occur as a result of the rodlike conformation of the molecules, but are not the driving force for the transition [14]. Solutions of isolated rod molecules should therefore be observed for low enough polymer concentrations. The corresponding concentration upper limit depends on the weight average molecular weight and polydispersity of the macromolecules : intermolecular interactions between rods should be negligible. The most stringent condition is that the polymer density should be lower than the inverse of the average excluded volume associated to a rod [46]. For monodisperse chains, the critical concentration is then :

$$
c_{\mathrm{p}}=\frac{6 M_{\mathrm{L}}^{3}}{\mathcal{N} \pi} \frac{1}{M^{2}}
$$

where $M_{\mathrm{L}}$ is the mass per unit contour length and $M$ the molecular weight of the polymers. $c_{p}$ is also related to the percolation threshold of the rods [15]. For polydisperse chains, we now approximate the molar mass distribution by the Schulz function [47].

$$
\begin{array}{r}
w(M)=\frac{M^{1 / U}}{\Gamma(1+1 / U)\left(\frac{M_{\mathrm{w}}}{1+1 / U}\right)^{1+1 / U}} \times \\
\quad \times \exp \left\{-\frac{M}{M_{\mathrm{u}}}(1+1 / U)\right\}
\end{array}
$$


where $\Gamma$ is the complete gamma function, $U=\left(M_{\mathrm{w}} / M_{\mathrm{N}}\right)-1$ characterizes the sample polydispersity, and $M_{\mathrm{N}}$ and $M_{\mathrm{w}}$ are the number and weight average molecular weights, respectively. Equation (13) is then replaced by:

$$
c_{\mathrm{p}}(U)=\frac{6 M_{\mathrm{L}}^{3}}{\mathcal{N} \pi} \frac{(1+U)^{2}}{(1+2 U)(1+3 U)} \frac{1}{M_{\mathrm{w}}^{2}} .
$$

In this section, we report light and neutron scattering experiments from $\mathrm{P}-4 \mathrm{BCMU}$ red solutions in protonated and perdeuteriated toluene with $c<c_{\mathrm{p}}(U)$ in conditions where isolated rod molecules could be observed, if they exist.

5.1 LIGHT SCATTERING FROM HIGH MOLECULAR WEIGHT P-4BCMU SOLUTIONS IN TOLUENE AT ROOM TEMPERATURE. - Combined static and dynamic light scattering experiments were performed at $20^{\circ} \mathrm{C}$, from red solutions of P-4BCMU-1 in protonated toluene for concentrations as low as $0.47 \times 10^{-3} \mathrm{~g} \mathrm{l}^{-1}$. The corresponding critical concentration $c_{\mathrm{p}}(U)$ is given in table IV. The condition

Table IV. - Critical concentrations related to the percolation threshold of rods.

\begin{tabular}{|c|c|c|c|}
\hline Sample & $\begin{array}{c}M_{\mathrm{w}} \\
\left(\mathrm{g} \mathrm{mole}^{-1}\right)\end{array}$ & $\begin{array}{c}U= \\
\left(M_{\mathrm{w}} / M_{\mathrm{N}}\right)-1\end{array}$ & $c_{\mathrm{p}}(U)\left(\mathrm{g} \mathrm{l}^{-1}\right)$ \\
\hline P-4BCMU-1 & 1670000 & 1.5 & $0.34 \times 10^{-3}$ \\
\hline P-4BCMU-2 & $\sim 43200$ & 1.5 & $\sim 0.51$ \\
\cline { 3 - 4 } & & 3 & $\sim 0.41$ \\
\hline
\end{tabular}

The critical concentrations $c_{\mathrm{p}}(U)$ are obtained from equation (15). For P-4BCMU-2, the value corresponding to $U=3$ is given in order to show the influence of polydispersity on the critical concentration.

$c<c_{\mathrm{p}}(U)$ is not fulfilled but the lower concentrations investigated here are much below a limit, proposed in the literature, for observation of red solutions of isolated $\mathrm{P}-4 \mathrm{BCMU}$ rods having approximately the same molecular weight : $0.01 \mathrm{~g} \mathrm{l}^{-1}$ [13].

The static light scattering results are shown in figure 9 as a plot of $K \mathrm{c} / R_{\theta}$ versus $q^{2} . K$ has been given earlier [6] and characterizes the contrast between the macromolecules and the solvent. $R_{\theta}$ is the Rayleigh ratio. Although there remains some uncertainty in determining these very low concentrations by optical absorption, the Zimm extrapolation method [30] yields an average molecular weight $M_{\mathrm{w}}$ of the order of $50 \times 10^{6} \mathrm{~g} \mathrm{~mole}^{-1}$. As compared to that of one P-4BCMU-1 chain, this result proves an aggregated structure, each particle including about 30 macromolecules. The corresponding average radius of gyration is independent of concentration in the investigated c-range $\left\langle R_{\mathrm{G}}^{2}\right\rangle_{z}^{1 / 2}=1150 \AA$. This value is much smaller than one would expect from a fully extended P-4BCMU chain. It is noteworthy that the radius of gyration does not depend on the refractive index increment.

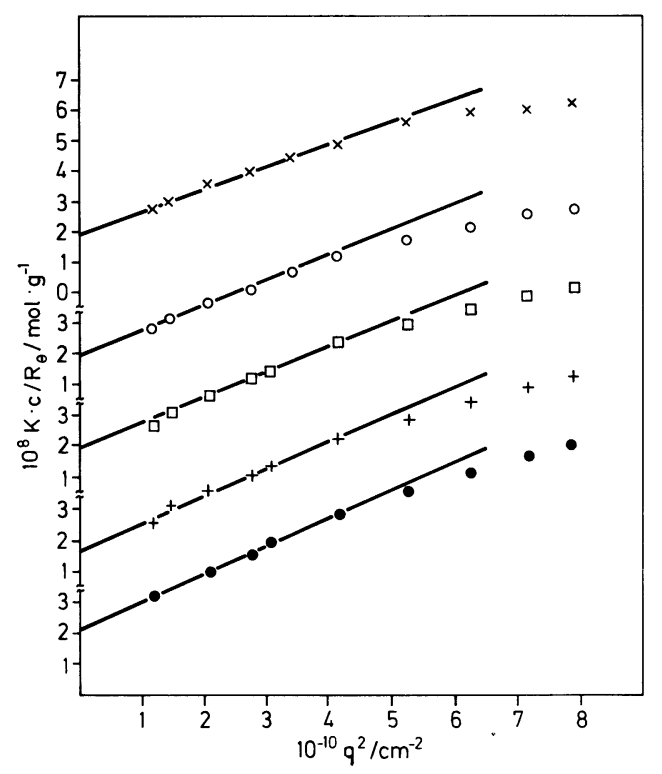

Fig. 9. - Reduced scattering intensity $k_{c} / R_{\Theta}$ versus $q^{2}$ for different concentrations of P-4BCMU in toluene at $20{ }^{\circ} \mathrm{C}: c=0.0023 \mathrm{mg} / \mathrm{ml}(+), 0.0037 \mathrm{mg} / \mathrm{ml}(\bullet), 0.0021$ $\mathrm{mg} / \mathrm{ml}(\square), 0.00047 \mathrm{mg} / \mathrm{ml}(\times), 0.00064 \mathrm{mg} / \mathrm{ml} \mathrm{(O)}$.

A correction could solely become necessary by considering a large anisotropy of the chain segments parallel and perpendicular to the chain axis. Such a correction is only important for rigid rods and can be written as [48]

$$
R_{\text {Gapp }}^{2}=R_{\mathrm{G}}^{2}\left\{1-\frac{4}{5} \mu+\frac{20}{35} \mu^{2}\right\}
$$

where $R_{\mathrm{Gapp}}^{2}$ and $R_{\mathrm{G}}^{2}$ are the measured and real radii of gyration ; $\mu=(\alpha-\beta) /(\alpha+2 \beta)$, the optical anisotropy expressed in terms of the axial polarizabilities $\alpha$ and $\beta$, along and perpendicular to the chain axis. In the limited cases of $\alpha=0(\beta \neq 0)$ and $\beta=0(\alpha \neq 0)$, the correction then amounts to at most $55 \%$ and $23 \%$, respectively.

The dynamic light scattering results are shown in figure 10 as a plot of $\Gamma / q^{2}$ versus $q^{2} . \Gamma$ is the first cumulant of the time correlation function. It was obtained from short time measurements and $\Gamma / q^{2}$ is the effective diffusion coefficient $D_{\text {app }}$. As expected for large, non-spherical particles, $\Gamma / q^{2}$ depends on the scattering vector and accordingly was extrapolated to zero $q$. This yields the translational diffusion 


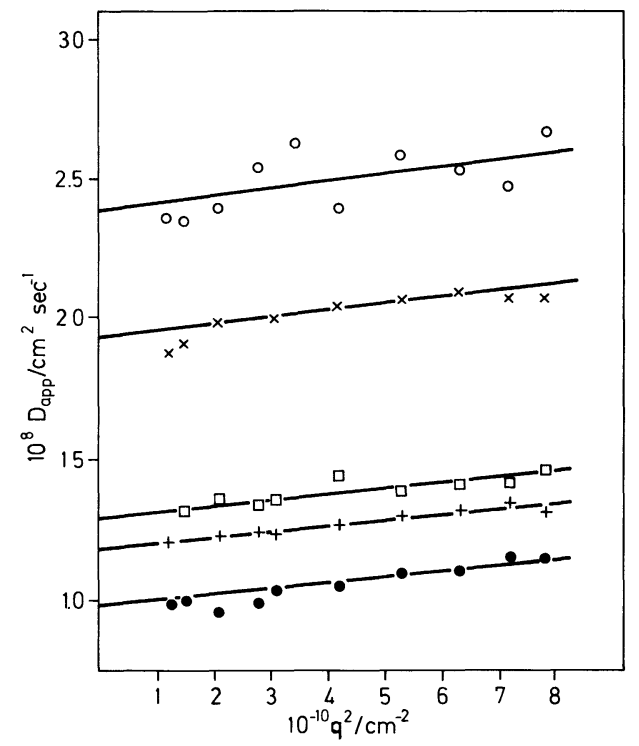

Fig. 10. - Reduced first cumulant $\Gamma / q^{2}=D_{\text {app }}$ versus $q^{2}$ for different concentrations of P-4BCMU in toluene at $20{ }^{\circ} \mathrm{C}: c=0.0023 \mathrm{mg} / \mathrm{ml}(\bullet), 0.0037 \mathrm{mg} / \mathrm{ml}(+), 0.0021$ $\mathrm{mg} / \mathrm{ml}(\square), 0.00047 \mathrm{mg} / \mathrm{ml}(\times), 0.00064 \mathrm{mg} / \mathrm{ml}(\mathrm{O})$.

coefficient $D_{z}$ or the hydrodynamic radius $R_{\mathrm{h}}=$ $\left\langle 1 / R_{\mathrm{H}}\right\rangle_{z}^{-1}$ via Stokes law :

$$
D_{z}=\frac{k_{\mathrm{B}} T}{6 \pi \eta_{\mathrm{s}}}\left\langle\frac{1}{R_{\mathrm{H}}}\right\rangle_{z},
$$

where $\eta_{\mathrm{s}}$ is the solvent viscosity.

The concentration dependent measurements of $R_{\mathrm{h}}$ exhibited a drastic increase of $R_{\mathrm{h}}$ with increasing concentrations. The data scatter considerably and do not allow to fit unambiguously a curve. This originates from the fact that even after centrifugation a few very large particles are present in the solutions which enter from time to time the scattering volume, thus disturbing the correlation function.

Moreover, the hydrodynamic radius of gyration is always significantly larger than the corresponding static radius of gyration as shown in figure 11 . This different behavior of $\left\langle 1 / R_{\mathrm{H}}\right\rangle_{z}^{-1}$ and $\left\langle R_{\mathrm{G}}^{2}\right\rangle_{z}^{1 / 2}$ is not easily explained and currently we can only offer qualitative and incomplete attempts at interpretation, all based on the picture of aggregated structures of polydiacetylenes as proposed earlier.

There could exist a densely packed and ordered center consisting of several P-4BCMU chains with some outgoing long flexible ends. These flexible ends could be low in mass and accordingly do not contribute much to the radius of gyration but they will cause a large hydrodynamic resistance of the structure. This is the fringed micelles model [17]. Nevertheless the main result of this study is that aggregated structures are observed even for concentrations as low as $0.5 \times 10^{-3} \mathrm{~g} \mathrm{l}^{-1}$ with high molecular weight P-4BCMU red solutions in toluene.

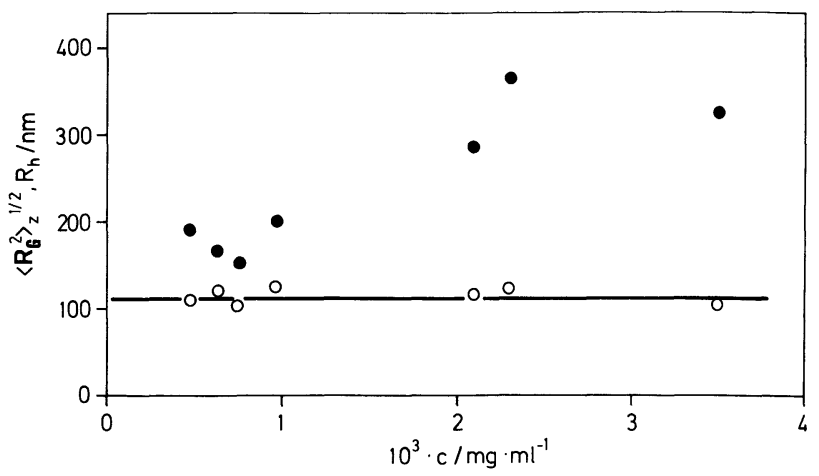

Fig. 11. - Plot of the radius of gyration $\left\langle R_{\mathrm{G}}^{2}\right\rangle_{z}^{1 / 2}(0)$ and of the hydrodynamic radius $\left\langle 1 / R_{\mathrm{H}}\right\rangle_{z}^{-1}(\bullet)$, as evaluated from measurements shown in figures 9 and 10, against the concentrations $c$.

5.2 NEUTRON SCATTERING FROM LOW MOLECULAR WEIGHT P-4BCMU SOLUTIONS IN PERDEUTERIATED TOLUENE. - The dilute regime can be reached at the concentrations needed in neutron scattering experiments only if $c_{\mathrm{p}}(U)$ is very much increased. Equation (15) shows that this requires smaller molecular weight, so short P-4BCMU chains prepared from P-4BCMU-1 by sonication as described in 2.1 were studied. Low molecular weight solutions in perdeuteriated toluene show a drastic color change when cooled below $T=55^{\circ} \mathrm{C}$. This color transition will be discussed elsewhere [42]. We are here only concerned with a search for isolated rods in the red solutions, so only two temperatures are considered : $T=70.5^{\circ} \mathrm{C}$ and $40.5^{\circ} \mathrm{C}$, corresponding to the yellow and red phases, respectively; and a restricted $q$-range is investigated : $0.003<q<$ $0.023 \AA^{-1}$. The macromolecules are referred to as $\mathrm{P}$ 4BCMU-2 in table I. They were characterized by light and neutron scattering since it must be shown first that $\mathrm{P}-4 \mathrm{BCMU}-1$ and -2 have the same average conformation. These techniques lead to nearly identical results, so only the neutron scattering experiments from yellow solutions of these polymers will be discussed.

5.2.1 Characterization of the low molecular weight P-4BCMU-2 chains. - Two solutions of P-4BCMU2 in perdeuteriated toluene were studied at $T=$ $70.5^{\circ} \mathrm{C}$. The concentrations were $c=0.47 \mathrm{~g} \mathrm{l}^{-1}$ and $1 \mathrm{~g} \mathrm{l}^{-1}$. Since they yield identical scattering functions $g(q, c)$ defined by equation (10) for $q>0.004 \AA^{-1}$, intermolecular correlations can be neglected in the corresponding $q$-range and $g(q, c)$ can be taken as the form factor $g(q)$. At small $q$ values, using the Zimm representation, $g(q)$ may be expanded as :

$$
\frac{1}{g(q)}=\frac{m}{M_{\mathrm{w}}}\left\{1+\frac{q^{2}\left\langle R_{\mathrm{G}}^{2}\right\rangle_{z}}{3}+O\left(q^{4}\right)\right\}
$$

and the corresponding curve is shown in figure 12 . 


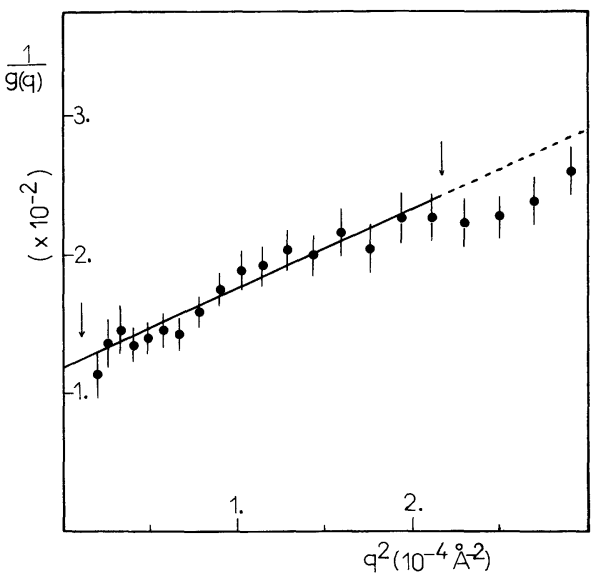

Fig. 12. - Zimm plot of the form factor of one P-4BCMU2 chain in perdeuteriated toluene at $T=70.5^{\circ} \mathrm{C}$. Arrows indicate the $q$-range used to fit the curve by expansion (17). It corresponds to $q^{2}\left\langle R_{\mathrm{G}}^{2}\right\rangle_{z}<3$.

The downwards curvature observed at high $q$ values suggests that polydispersity is larger than or comparable to $U=1$, proving that the Zimm representation is well adapted to our data [49]. Indeed we may argue that equation (17) is valid for $q^{2}\left\langle R_{\mathrm{G}}^{2}\right\rangle_{z}<3$ as long as $U<2$. GPC measurements made in Mainz indicate that the polydispersity of the P-4BCMU-2 sample is nearly the same as that of the P-4BCMU-1 source sample $U \sim 1.5$. Thus $M_{\mathrm{w}}=43200 \mathrm{~g} \mathrm{~mole}^{-1}$ $\AA^{-1}$ (Tab. IV) and $\left\langle R_{\mathrm{G}}^{2}\right\rangle_{z}^{1 / 2}=121 \AA$. The corresponding contour lengths are $L_{\text {trans }} \sim 400 \AA$ and $L_{\text {cis }} \sim 300 \AA$. Polydispersity is not the only source of uncertainty on $M_{\mathrm{w}}$ : chemical defects, for instance missing side-groups in P-4BCMU-2, change the contrast factor.

The average radius of gyration $\left\langle R_{\mathrm{G}}^{2}\right\rangle_{z}^{1 / 2}$ obtained does not correspond to that of a fully extended conformation. Indeed for rigid rods $\left\langle R_{\mathrm{G}}^{2}\right\rangle_{z}$ is [50] :

$$
\left\langle R_{\mathrm{G}}^{2}\right\rangle_{\mathrm{z}}=\frac{(1+2 U)(1+3 U)}{(1+U)^{2}} \frac{M_{\mathrm{w}}^{2}}{12 M_{\mathrm{L}}^{2}}
$$

so $121 \AA$ would correspond to $U<0.1$ if macromolecules are in the trans configuration of the double bonds ; and $U<0.4$ for the cis isomeric state. Such small polydispersities are in contradiction with the downwards curvature noted in figure 12 at higher $q$ values. On the other hand, in the wormlike chain model, $\left\langle R_{\mathrm{G}}^{2}\right\rangle_{z}$ is given by [50] :

$$
\begin{aligned}
& \left\langle R_{\mathrm{G}}^{2}\right\rangle_{z}=\frac{1+2 U}{1+U} \frac{M_{\mathrm{w}}}{M_{\mathrm{L}}} \frac{b}{6}\left\{1-\frac{1+U}{1+2 U} \frac{M_{\mathrm{L}}}{M_{\mathrm{w}}} \frac{3 b}{2} \times\right. \\
& \quad \times\left(1-\frac{M_{\mathrm{L}}}{M_{\mathrm{w}}} b\right)-\frac{(1+U)^{2}}{1+2 U}\left(\frac{M_{\mathrm{L}}}{M_{\mathrm{w}}}\right)^{3} \frac{3 b^{3}}{4} \\
& \left.\quad \times\left[1-\left(1+\frac{U}{1+U} \frac{M_{\mathrm{w}}}{M_{\mathrm{L}}} \frac{2}{b}\right)^{-1 / U}\right]\right\}
\end{aligned}
$$

where $b$ is the Kuhn statistical length, i.e. twice the persistence length. This relation between $\left\langle R_{\mathrm{G}}^{2}\right\rangle_{z}$ and $b$ is shown in figure $13 . U=1.5$ yields $b_{\text {trans }}=$ $200 \pm 20 \AA$. To recover the long chain statistical length of $280 \AA$ one would need $U \sim 0.5$. If one assumes that, contrary to the long chains of $\mathrm{P}$ 4BCMU-1, short P-4BCMU-2 chains are cis, $U=1.5$ leads to $b_{\text {cis }} \sim 400 \AA>L_{\text {cis }}$; an increased rigidity does not seem reasonable, so a cis configuration is unlikely.

Thus, short chains should be less stiff than longer ones, either intrinsically or because defects are
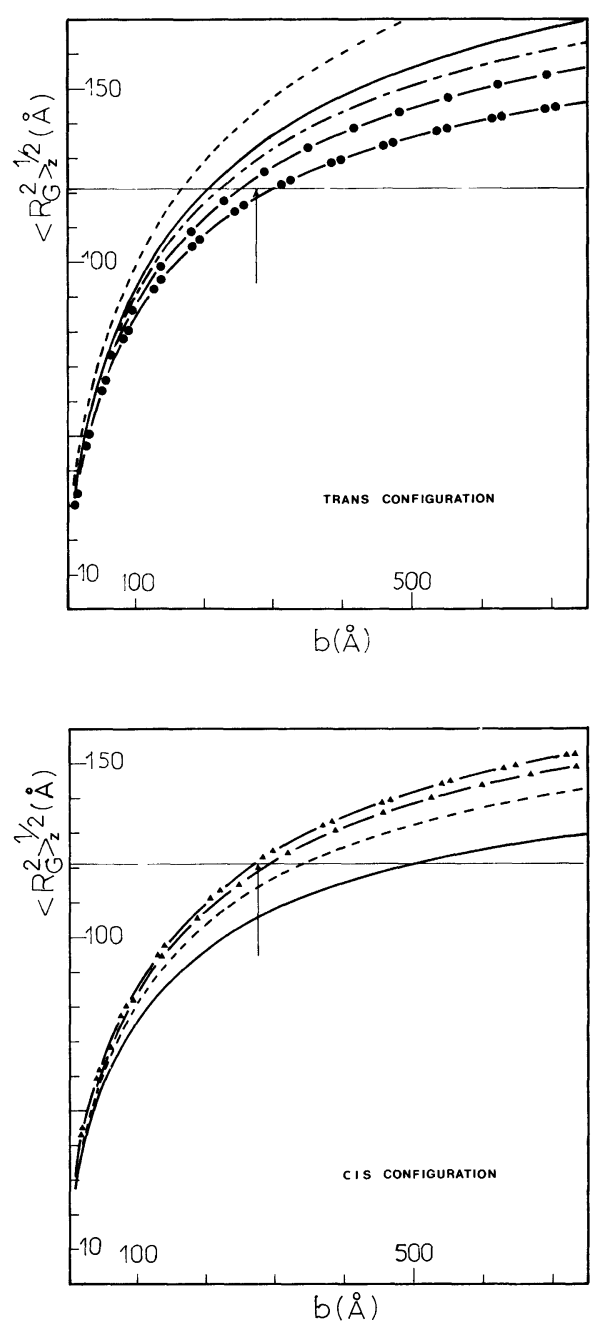

Fig. 13. - Plot of the radius of gyration $\left\langle R_{\mathrm{G}}^{2}\right\rangle_{z}^{1 / 2}$ versus the statistical length $b$ as evaluated from relation (19) using the $M_{\mathrm{w}}$ value obtained from figure 12 , for various polydispersities : $U=0.4(-),. 0.6(-),. 0.8(--), 1(-), 2$ $(--), 3(-\Delta)$, and $4(-\Delta \mathbf{\Delta})$. The $\left\langle R_{\mathrm{G}}^{2}\right\rangle_{z}^{1 / 2}$ value obtained from figure 12 is shown (horizontal line) and the $b$ value measured for the corresponding long chains at $T=70^{\circ} \mathrm{C}$ is indicated by an arrow : a) assuming the trans configuration of the double bonds $\left(M_{\mathrm{L}} \sim 100 \mathrm{~g}\right.$ mole $\left.\AA^{-1} \AA^{-1}\right)$; b) assuming the cis configuration $\left(M_{\mathrm{L}} \sim\right.$ $\left.140 \mathrm{~g} \mathrm{~mole}^{-1} \AA^{-1}\right)$. 
introduced by the sonication process. In either case, they should also be less conjugated. Indeed, their yellow spectrum is slightly different, the wavelength of maximum absorption in P-4BCMU-2 being blueshifted by $80 \AA$ in comparison to P-4BCMU-1 at the same temperature.

Finally, all the observations are consistent with the short P-4BCMU-2 chains being, as long chains are, wormlike chains in the trans configuration of the double bonds, but with a smaller statistical length of about $200 \AA$ at $T=70{ }^{\circ} \mathrm{C}$. Since the yellow-red optical transition follows the same equation (11) as the long chains do, but at lower temperatures and on a broader range (between 54 and $44^{\circ} \mathrm{C}$ ) that is with a different $X(T)$, P-4BCMU2 can be used to investigate the existence of isolated red rods, and it will be possible to extrapolate to the long chain sample P-4BCMU-1 and P-4BCMU's in general.

5.2.2 Neutron scattering from low molecular weight $P-4 B C M U$ red solutions in perdeuteriated toluene. Table IV shows that $c<c_{\mathrm{p}}(U)$ assuming reasonable values of $U$, for the $c=0.47 \mathrm{~g}^{-1}$ P-4BCMU-2 solution in perdeuteriated toluene. This solution exhibits on cooling a yellow to red color transition similar to that studied in Section 4 ; it occurs around $54{ }^{\circ} \mathrm{C}$, with a transition range from 54 to $44^{\circ} \mathrm{C}$. It then differs somewhat for the long chain behavior shown in figure 6 [38]. The yellow and red phases were therefore studied at 70.5 and $40.5^{\circ} \mathrm{C}$ respectively. The coherent differential cross sections $\Sigma^{\mathrm{coh}}(q)$ of this solution are shown in figure 14 for both temperatures. Obviously aggregation occurs in the red phase of P-4BCMU-2.

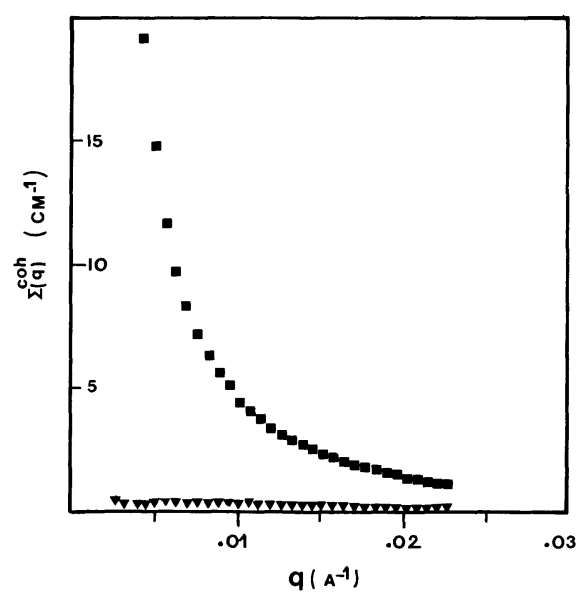

Fig. 14. - Coherent differential cross sections $\Sigma^{\text {coh }}(q)$ of a $c=0.47 \mathrm{gl}^{-1}$ P-4BCMU-2 solution in perdeuteriated toluene at two temperatures : $T=70.5^{\circ} \mathrm{C} \quad(\nabla)$ and $T=40.5^{\circ} \mathrm{C}(\square)$.

In another series of experiments, which will be reported elsewhere [42], the scattering function of solutions of the same polymer was measured as a function of temperature, showing that, on increasing $T$, aggregation disappears abruptly at $69^{\circ} \mathrm{C}$, coinciding with the red to yellow color transition found optically. Two kinds of scattering curve modifications are observed, suggesting once more a twostep process for aggregation.

\section{Conclusion.}

6.1 CONFORMATION IN GOOD SOLVENT AND ORIGIN OF THE RIGIDITY. - In good solvents, P-3BCMU and P-4BCMU dissolve with a yellow color and form semi-rigid coils with similar average geometry.

On spatial scales where the side group finite size can be neglected, the internal structure of these coils may be described by the continuous wormlike chain model. The macromolecules are then well represented by thin threads and their stiffness is characterized by a persistence length close to $160 \AA$ at room temperature. Viscosity measurements give the same value for P-TS12, a polydiacetylene whose side groups cannot form hydrogen bonds [6]. A theoretical calculation, considering a single rotational isomer undergoing twisting and bending fluctuations and neglecting side groups also yielded nearly the same persistence length [43]. This shows that the conjugated structure of the backbone is the main cause for the rigidity of polydiacetylenes in good solvents. The persistence length variation with $T$ : between 295 and $360 \mathrm{~K}$ is compatible with the $T^{-1}$ dependence expected for a wormlike chain. Simultaneously, a progressive blue-shift of the absorption spectrum upon increase of $T$ is observed [38], in agreement with a relation between the average geometry of the chain and its electronic structure [51]. Finally the mass per unit contour length of the thin thread is found in agreement with the trans configuration of the double bonds.

The average side group conformation was not studied in detail ; a more accurate description would require the use of selectively labeled side groups and larger $q$ values. Nevertheless, the results are compatible with a model of a wormlike ribbon containing the inner part of side groups, the outer one being more disordered.

6.2 THE WORMLIKE versus DISCRETE DEFECTS CHAIN MODEL. - The electronic properties of the conjugated carbon chain of polydiacetylenes in solution, particularly the absorption spectrum, have been usually discussed up to now in terms of short rigid fully conjugated and electronically decoupled platelets of variable length $[1,7,8]$. Is this compatible with a wormlike chain conformation? Continuous curvature is a mathematically convenient limit, but rigid segments of at least the length of a carboncarbon bond, certainly exist. If such segments are 
short enough, the difference with a continuously curved thread may be too small to modify measurably the scattering function.

The wormlike model implies that successive rigid segments are not freely jointed: the angle they make cannot take any value with equal probability. Relaxing this assumption leads to different models of semi-rigid chains. It is shown in the appendix that models of chains made of freely jointed rods lead, for the same radius of gyration, to different scattering functions (due to differences in chain compactness), and that only the wormlike chain prediction agrees with experiment. Thus, whatever the defects leading to curvature of polydiacetylene chains are, the orientations of the chain on either side of the defect are correlated.

In the Porod-Kratky model of a chain made of short, correlated, rigid segments of equal length, the angle $\phi$ between successive segments is related to their length $l$ through [31] :

$$
\cos \phi=1-2 l / b
$$

$b$ being twice the persistence length.

In polydiacetylenes, it is reasonable to assume that the shortest possible rigid segment is a repeat unit of length $a \sim 5 \AA$, corresponding to an average angle $\phi \sim 14.8$ degrees : this is the smallest possible average misorientation compatible with the present neutron scattering data. The misorientation increases rapidly with $l$. For $l=4 a, \phi=30$ degrees, and for $l=7 a, \phi=40$ degrees. These values put a constraint on the type of defects compatible with the average conformation determined here.

This problem has been briefly discussed elsewhere [51], where it was pointed out that very frequent misorientations, with an average angle given by (20), could effectively localize the electrons along the onedimensional chain, over a distance much larger than $a$, and which would have no reason to be an integral multiple of $a$. This would explain the total absence of structure in the visible absorption spectra, which is difficult to account for in the discrete defects model [8].

In conclusion, the scattering data are compatible only with the chain being made of slightly misoriented short segments, one or a few units long: that is, a wormlike chain, if not a strictly continuously curved thread.

6.3 THE COLOR TRANSITION. - In all cases studied here, the color transition for P-4BCMU solutions in toluene occurs simultaneously with aggregation. No red solution of isolated macromolecules has been observed even for concentrations below the critical values associated with an overlap of rod molecules. Thus, either the change in the electronic structure corresponding to the absorption redshift is produced by aggregation; or, if it is due to an intramolecular coil to rod conformational change, the rods are insoluble and immediately form aggregated structures. This is a matter of current debate that present data alone cannot settle [52]. A cis $\rightarrow$ trans isomerization cannot explain the yellow $\rightarrow$ red color transition since the macromolecules are in the trans configuration of the double bonds in the yellow phase.

One unambiguous experimental result is the almost concentration independent temperature at which the color transition occurs [see for instance 1, $4,44]$. This fact can be explained in different ways :

a) the chains form amorphous aggregates, because the solution is cooled below the $\theta$-temperature. For dilute polymer solutions, the $\theta$-temperature is known not to depend on polymer concentration;

b) if the chains partially crystallize, we have to distinguish between a thermal and an athermal nucleation. For the unlikely case of thermal nucleation, one indeed should observe a concentration dependent crystallization temperature. However, in practically all cases where nucleation of polymer crystals have been investigated, athermal nucleation (caused by impurities) is observed, in which case no concentration dependence of the crystallization temperature is expected. Thus, the concentration independent transition temperature does not rule out the occurrence of an aggregation and/or crystallization process and accordingly does not prove the intramolecular coil-to-rod transition to cause the color change.

The structure of the aggregates has not been studied in details yet and that of the macromolecules inside the aggregates remains to be investigated. No self similar structure for the gel has been observed. Rather, our results would agree with aggregates having a well-defined geometry, for instance a fringed micelle. The aggregation seems also to proceed in two steps. Indeed, by decreasing the temperature, two kinds of scattering behavior modifications are observed. The first is related to the optical transition and occurs below $66^{\circ} \mathrm{C}$ in a $T$ range which depends on the weight average molecular weight and polydispersity : the macromolecules form clusters. The second is observed when the optical transition is completed. Cluster-cluster aggregation is a possibility. An ordering of aggregates has been suggested theoretically [53]. However, we see no anisotropy of the scattering. Finally, although clusters formation is fast with regard to the time scale of the scattering experiments, cluster-cluster aggregation should be a slower process. This will be discussed elsewhere.

\section{Acknowledgments.}

It is a pleasure for us to thank B. Cabane and R. C. Oberthür for valuable discussions. 
Appendix.

Asymptotic scattering behaviors of linear semi-rigid macromolecules according to continuous and discrete defect models.

The structure of linear semi-rigid macromolecules is mainly described by the wormlike and zig zag chain models. In the first model, the shape of molecules is approximated by a thin thread. The flexibility, which is primarily due to rotational isomerisms, is then caused by curvature fluctuations. In the second one, a polymer is represented by freely linked rods and the flexibility results from sharp bends. In any case, chain cross section as well as excluded volume effects are neglected. Here, we wish to compare the related chain structure factors, at the same mean square radius of gyration $R_{\mathrm{G}}^{2}$. At low resolution, the previous pictures are identical for long enough chains and, for $q R_{\mathrm{G}}<1$, the form factor $P(q)=$ $S(q) / S(0)$ is :

$$
\frac{1}{P(q)}=1+\frac{q^{2} R_{\mathrm{G}}^{2}}{3}+O\left(q^{4}\right) .
$$

For a contour length $L \gg b$, this implies the same Kuhn statistical length $b$ defined as [33] :

$$
b=\frac{6 R_{\mathrm{G}}^{2}}{L} \text {. }
$$

For a wormlike chain, $b$ is twice the persistence length defined by equation (3). For a regular broken chain, $b$ represents the length of the rods. Finally, in the statistical zig zag model, $b$ is twice the average length of rods [A.1]. In this case, long chains with a small number of defects are required as the distributions of rod lengths are assumed to be independent with a characteristic exponential form :

$$
p(l)=\frac{2}{b} \exp \left\{-\frac{2 l}{b}\right\} \text {. }
$$

Thus, in this appendix, the continuous model should not be regarded as a limiting case of the discrete defect model.

The form factor of an infinite wormlike chain has been calculated by des Cloizeaux [34], while, for $0.05<L / b<10^{4}$ and $q b>10$, an interpolation formula has been given by Yoshizaki and Yamakawa [A.2]. The form factor of a broken chain formed by rods of equal length $b$ has been obtained by Hermans and Hermans [A.3]. It can be written as :

$$
\begin{aligned}
\frac{L}{b} P(q)=\frac{2}{x} & \left\{S_{i}(x)-\frac{1-\cos x}{x}\right\}+ \\
& +2 \frac{S_{i}^{2}(x)}{x^{2}(1-y)}\left\{1-\frac{1-y^{L / b}}{\frac{L}{b}(1-y)}\right\}
\end{aligned}
$$

with $x=q b, y=\frac{\sin q b}{q b}$ and $S_{i}(u)=\int_{0}^{u} \mathrm{~d} t \frac{\sin t}{t}$.
The form factor of a statistical broken chain formed by rods of average length $b / 2$ has been calculated by Skvortsov, Birshtein and Fedorov, using the assumptions mentioned above [A.1]. It can be written as :

$$
\frac{L}{b} P(q)=\frac{z}{1-z}\left\{1-\frac{b}{2 L} z^{2} \frac{1-z^{2 L / b}}{1-z}\right\}
$$

with $z=\frac{\tan ^{-1} q b}{q b}$.

These structure factors are presented in figure A.1, together with those of rods [A.4]. At small $q$ values, they are almost identical. Specifically, the form factor of a long regular broken chain corresponds to that of a long wormlike chain up to $q b \sim 6$. The differences between the three structural models are only observed at high $q$ values. The $q^{-1}$ scattering behavior of rod like molecules is found in any case, as demonstrated earlier [A.5]. However, the asymptotes of the form factors are different, and, in a Kratky plot as in figure A.1, do not pass through the same point on the $y$ axis though they have the same slope. Thus, the asymptotic behaviors are :

$$
\lim _{q \rightarrow \infty} \frac{L}{b} P(q)=\frac{\pi}{q b}+\frac{B}{(q b)^{2}}
$$

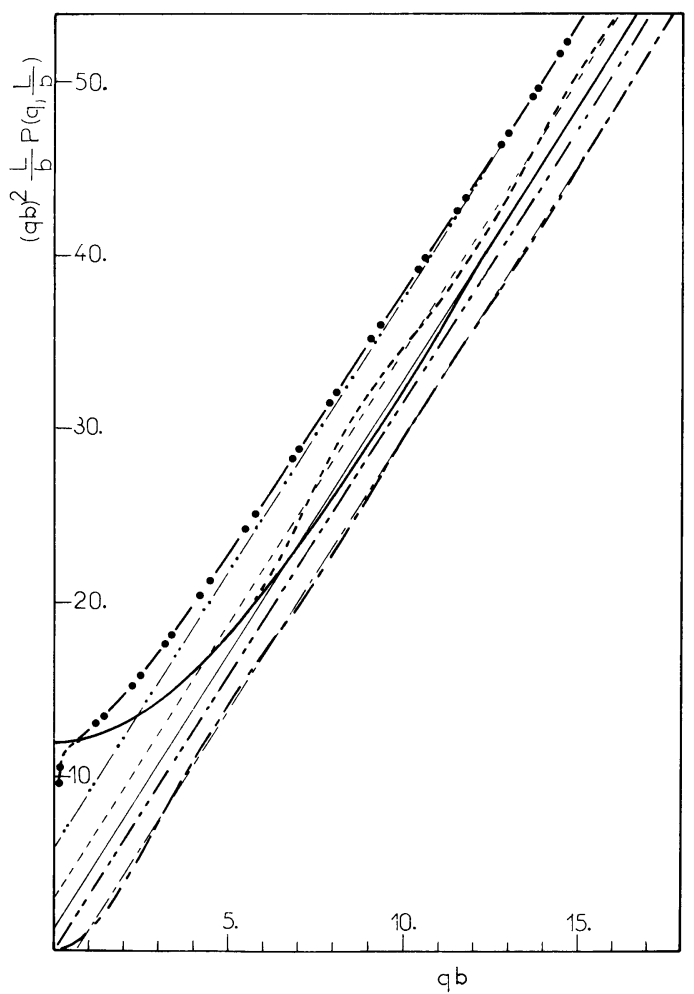

Fig. A.1. - Kratky plot of the structure factors and their asymptotic forms for several models : the infinitely long wormlike chain (-), the regular broken chain for $L / b=10^{4}(--)$, the statistical broken chain for $L / b=10^{4}$ (-..), the infinite rod (- --) and a short rod with $L$ equal to the $b$ value of the chains in the other models $(--) . L$ is the contour length and $b$ the statistical length. 
and $B$ depends on the model :

$$
\begin{aligned}
& B=\frac{4}{3} \text { for long enough wormlike chains } \\
& B=\frac{\pi^{2}-4}{2} \text { for the regular zig zag model } \\
& B=\pi^{2}-4 \text { for the statistical zig zag model } \\
& B=-\frac{2 b}{L} \text { for rods, i.e. } B=0 \text { for } \frac{L}{b} \gg 1
\end{aligned}
$$

Moreover the structure factor of a regular broken chain shows characteristic oscillations around its asymptote. Such oscillations are smoothed and disap- pear when statistical distributions of rod lengths are considered. Nevertheless, it should be emphasized that, for long stastistical broken chains, the $q^{-1}$ decay is observed beyond $q b \sim 7$ as for long wormlike chains.

At this stage, we can note that the constraint of universal links in the statistical zig zag model is not important at high $q$ values. Indeed, the differences in the asymptotic behaviors (A.6) can be explained by the differences in the compactness for the internal structures of the related chains. Therefore the results corresponding to the statistical zig zag model hold as long as defects can be considered as sharp bends.

\section{References}

[1] Patel, G. N., A.C.S.-Polym. Chem. Div., Polym. preprints 19 (1978) 154 ;

Patel, G. N., Chance, R. R., Witt, J. D., J. Polym. Sci. Polym. Lett. ed. 16 (1978) 607, J. Chem. Phys. 70 (1979) 4387.

[2] Wenz, G., Wegner, G., Makromol. Chem. Rapid Commun. 3 (1982) 231.

[3] Plachetta, C., Rau, N. O., Hauck, A., Schulz, R. C., Makromol. Chem. Rapid Commun. 3 (1982) 249 ;

Plachetta, C., Schulz, R. C., Makromol. Chem. Rapid Commun. 3 (1982) 815 ;

Plachetta, C., Rau, N. O., Schulz, R. C., Mol. Cryst. Liq. Cryst. 96 (1983) 141.

[4] Patel, G. N., Walsh, E. K., J. Polym. Sci. Polym. Lett. ed. 17 (1979) 203.

[5] Patel, G. N., Witt, J. D., Khanna, Y. P., J. Polym. Sci. Polym. Lett. ed. 18 (1980) 1383.

[6] Wenz, G., Müller, M. A., Schmidt, M., Wegner, G., Macromolecules 17 (1984) 837.

[7] Chance, R. R., Patel, G. N., Witt, J. D., J. Chem. Phys. 71 (1979) 206;

Chance R. R., Macromolecules 13 (1980) 396.

[8] Chance, R. R., Shand, M. L., Le Postollec, M., Schott, M., J. Polym. Sci. Polym. Lett. ed. 19 (1981) 529 ;

Shand, M. L., Chance, R. R., Le Postollec, M., Schotт, M., Phys. Rev. B 25 (1982) 4431.

[9] Berlinsky, A. J., Wudl, F., Lim, K. C., Fincher, C. R., Heeger, A. J., J. Polym. Sci. Polym. Phys. ed. 28 (1984) 847.

[10] Zimm, B. H., Bragg, J. K., J. Chem. Phys. 28 (1958) $1246 ; 31$ (1959) $526 ; 33$ (1960) 1349.

[11] Se, K., Ohnuma, H. Kotaka, T., Polym. J. 14 (1982) 895.

[12] Lim, K. C., Fincher, C. R., Heeger, A. J., Phys. Rev. Lett. 50 (1983) 1934 ;

Lim, K. C. Sinclair, M., Casalnuovo, S. A., Fincher, C. R., Wudl, F., HeEger, A. J., Mol. Cryst. Liq. Cryst. 105 (1984) 329.

[13] Lim, K. C., Heeger, A. J., J. Chem. Phys. 82 (1985) 522.
[14] Sinclair, M., Lim, K. C., Heeger, A. J., Phys. Rev. Lett. 51 (1983) 1768.

[15] Balberg, I., Anderson, C. H., Alexander, S., WAgner, N., Phys. Rev. B. 30 (1984) 3933 ;

Bug, A. L. R., Safran, S. A., Webman, I., Phys. Rev. Lett. 54 (1985) 1412.

[16] Müller, M. A., Schmidt, M., Wegner, G., Makromol. Chem. Rapid Commun. 5 (1984) 83.

[17] Müller, M. A., Thesis, University of Freiburg (1984).

[18] DE Gennes, P. G., Scaling Concepts in Polymer Physics (Cornell U. Press, New York) (1979) p. 186.

[19] Odell, J. A., Keller, A., Miles, M. J., Polym. Commun. 24 (1983) 7.

[20] Merrill, E. W., Horn, A. F., Polym. Commun. 25 (1984) 144.

[21] Müller, M. A., Wegner, G., Makromol. Chem. 185 (1984) 1727.

[22] OberthÜR, R. C., I.L.L. Internal Report ;

Ragnetti, M., Geiser, D., Höcker, H., OberTHÜR, R. C., Makromol. Chem. 186 (1985) 1701.

[23] van Krevelen, D. W., Properties of Polymers, (Elsevier Pub. Co., New York) 1972, chapter IV.

[24] Cotton, J. P., Benoit, H., J. Phys. France 36 (1975) 905.

[25] Koglin, W., Kurzes Handbuch Aus Chemie (Van den Hoeck and Ruprecht) 1951-1954.

[26] Riddick, J. A., Binger, W. B., Organic Solvents, 3rd edition (Wiley Interscience, New York) 1970.

[27] Bantle, M., Schmidt, M., Burchard, W., Macromolecules 15 (1982) 1604.

[28] Debye, P., J. Phys. Colloid Chem. 51 (1947) 18.

[29] Flory, P. J., Statistical Mechanics of Chain Molecules (J. Wiley and Sons, New York) 1969.

[30] Zıмm, B. H., J. Chem. Phys. 16 (1948) 1093 ;

Benoit, H., Benmouna, M., Polymer 25 (1984) 1059.

[31] Porod, G., Monatsh. Chem. 80 (1949) 251 ; 
Kratky, O., Porod, G., Rec. Trav. Chim. Pays-Bas 68 (1949) 1106.

[32] Landau, L. D., Lifshitz, E. M., Statistical Physics 3rd edition, (Pergamon Press) 1980, part 1, p. 396.

[33] Kunn, W., Kolloid Z. 76 (1936) 258 and 87 (1939) 3.

[34] Des Cloizeaux, J., Macromolecules 6 (1973) 403.

[35] Rawiso, M., Duplessix, R., Picot, C., Macromolecules 20 (1987) 630.

[36] Porod, G., in Small Angle X-Ray Scattering, Eds.

- O. Glatter, O. Kratky (Academic Press, New York) 1982, p. 17-51.

[37] KRATKY, O., Z. Electrochem. 60 (1956) 245 ;

Luzzati, V., Acta Crystallogr. 13 (1960) 939 ;

Koyama, R., J. Phys. Soc. of Jpn. 36 (1974) 1409.

[38] Aimé, J. P., Bargain, F., Fave, J. L., Schott, M., to be published.

[39] Guinier, A., Fournet, G., Small Angle Scattering of X-Rays (J. Wiley and Sons, New York) 1955.

[40] The exact form factor of a rigid sheet with zero thickness is

$$
\begin{aligned}
P(q)=\frac{2}{q L} & \left\{\frac{\pi}{q W}\left(\int_{0}^{q W} \frac{J_{1}(X)}{X} \mathrm{~d} X\right)+\right. \\
& \left.+\frac{1}{q L}\left(\frac{\sin (q W / 2)}{q W / 2}\right)^{2}-\frac{\sin q L}{(q L)^{2}}\right\}
\end{aligned}
$$

where $J_{1}$ is the Bessel function of order $1 ; L$, the length of the sheet and $W$, its width. For nonzero thickness, this expression would be multiplied by an exponential term.

[41] Holtzer, A. M., J. Polym. Sci. 17 (1955) 432.

[42] Aimé, J. P., Rawiso, M., Sснотt, M., to be published.

[43] Allegra, G., Brückner, S., Schmidt, M., Wegner, G., Macromolecules 19 (1985) 399.

[44] Aimé, J. P., Fave, J. L., Schott, M., Europhys. Lett. 1 (1986) 505.

[45] Chance, R. R., Washabaugh, M. W., Hupe, D. J., in Polydiacetylenes, Eds. D. Bloor and R. R. Chance, NATO ASI E 102 (1985) 239.
[46] Onsager, L., Ann. N.Y. Acad. Sci. 51 (1949) 627.

[47] Schulz, G. V., Z. Phys. Chem. B 43 (1939) 25.

[48] Benort, H., in Light Scattering from Dilute Polymer Solutions, Eds. D. McIntyre and F. Gornick, 1964, p. 295 ;

Horn, P., Benoit, H., Oster, G., J. Chim. Phys. et Phys. Chim. Biol. 48 (1951) 1.

[49] Kirste, R. G., Oberthür, R. C., in Small Angle XRay Scattering, Eds. O. Glatter, O. Kratky (Academic Press) 1982, p. 387-431.

[50] Oberthür, R. C., Makromol. Chem. 179 (1978) 2693.

[51] Aimé, J. P., Rawiso, M., SchotT, M., in Electronic properties of polymers, Eds. H. Kuzmany, M. Mehring and S. Roth (Springer) 1987.

[52] We are most grateful to one of the referees for bringing to our attention the following reference :

Rughooputh, S. D. D. V., Hotta, S., Heeger, A. J., Wudl, F., J. Polym Sci. Polym. Phys. Ed. 25 (1987) 1071

in which the question of the intramolecular origin of such chromic transitions is discussed in general terms, and with special reference to polythienylenes.

[53] Halperin, A., J. Chem. Phys. 85 (1986) 1081.

[A1] Skvortsov, A. M., Birshtein, T. M., Fedorov, B. A., Dokl. Akad. Nauk SSSR 182 (1968) 322; Sov. Phys. Dokl. 13 (1969) 916.

[A2] Yoshizaki, T., YamaKaWa, H., Macromolecules 13 (1980) 1518.

[A3] Hermans, J., Hermans, J. J., J. Phys. Chem. 62 (1958) 1543.

[A4] Neugebauer, T., Ann. Phys. 42 (1943) 509.

[A5] Luzzati, V. Benoit, H., Acta Crystallogr. 14 (1961) 297 ;

Sadron, C., J. Chim. Phys. et Phys. Chim. Biol. 58 (1961) 877. 